ARTICLE

\title{
The phosphoinositide coincidence detector Phafin2 promotes macropinocytosis by coordinating actin organisation at forming macropinosomes
}

\author{
Kay Oliver Schink (10 1,2凶 , Kia Wee Tan (10 1,2, Hélène Spangenberg ${ }^{1,2}$, Domenica Martorana ${ }^{1,2}$, Marte Sneeggen ${ }^{1,2}$,
} Virginie Stévenin (10 ${ }^{3,4}$, Jost Enninga ${ }^{4}$, Coen Campsteijn (1) ${ }^{5}$, Camilla Raiborg (1) ${ }^{1,2} \&$ Harald Stenmark (1) $1,2 \otimes$

Uptake of large volumes of extracellular fluid by actin-dependent macropinocytosis has an important role in infection, immunity and cancer development. A key question is how actin assembly and disassembly are coordinated around macropinosomes to allow them to form and subsequently pass through the dense actin network underlying the plasma membrane to move towards the cell center for maturation. Here we show that the PH and FYVE domain protein Phafin2 is recruited transiently to newly-formed macropinosomes by a mechanism that involves coincidence detection of Ptdlns3P and Ptdlns4P. Phafin2 also interacts with actin via its $\mathrm{PH}$ domain, and recruitment of Phafin2 coincides with actin reorganization around nascent macropinosomes. Moreover, forced relocalization of Phafin2 to the plasma membrane causes rearrangement of the subcortical actin cytoskeleton. Depletion of Phafin2 inhibits macropinosome internalization and maturation and prevents KRAS-transformed cancer cells from utilizing extracellular protein as an amino acid source. We conclude that Phafin2 promotes macropinocytosis by controlling timely delamination of actin from nascent macropinosomes for their navigation through the dense subcortical actin network.

\footnotetext{
${ }^{1}$ Centre for Cancer Cell Reprogramming, Faculty of Medicine, University of Oslo, Montebello, N-0379 Oslo, Norway. ${ }^{2}$ Department of Molecular Cell Biology, Institute for Cancer Research, Oslo University Hospital, Montebello, 0379 Oslo, Norway. ${ }^{3}$ Department of Cell and Chemical Biology, Leiden University Medical Center, Einthovenweg 20, 2333 ZC Leiden, The Netherlands. ${ }^{4}$ Institut Pasteur, Dynamics of Host-Pathogen Interactions Unit, 25 Rue du Dr. Roux, Paris, France. ${ }^{5}$ Department of Molecular Medicine, Institute of Basic Medical Sciences, University of Oslo, PO Box 1112 Blindern, 0317 Oslo, Norway.

凶email: Kay.Oliver.Schink@rr-research.no; h.a.stenmark@medisin.uio.no
} 
$\mathrm{M}$ acropinocytosis is an actin-dependent endocytosis mechanism that allows cells to take up extracellular fluids and soluble macromolecules by the formation of membrane ruffles that collapse into large vacuoles ${ }^{1}$. Macropinocytosis evolved early in evolution, probably as an uptake mechanism for nutrients in free-living organisms ${ }^{2}$. In mammalian cells, this mechanism is important in dendritic cells and macrophages which use it to sample body fluids for antigens. Macropinocytosis is exploited by pathogens, which trigger macropinocytosis in order to be taken up by host cells ${ }^{3}$. RAStransformed cancer cells exhibit high levels of macropinocytosis and exploit this mechanism to take up nutrients from the surrounding medium ${ }^{4}$.

Phosphoinositides are key regulators of macropinocytosis. PtdIns $(4,5) \mathrm{P}_{2}$ and $\operatorname{PtdIns}(3,4,5) \mathrm{P}_{3}$ localize to macropinosome cups, where they can trigger actin rearrangements by activating different actin-regulating pathways ${ }^{5,6}$. PtdIns $(3,4,5) \mathrm{P}_{3}$ localizes to the rim of macropinosome cups. After ruffle closure, relatively little is known about the role of phosphoinositides. A phosphatase cascade metabolizes PtdIns $(3,4,5) \mathrm{P}_{3}$ via $\operatorname{PtdIns}(3,4) \mathrm{P}_{2}$ and PtdIns3P to PtdIns ${ }^{7}$. Moreover, dephosphorylation of PtdIns $(4,5)$ $\mathrm{P}_{2}$ to PtdIns4P by OCRL has recently been shown to be important for macropinosome closure ${ }^{8}$.

After internalization, macropinosomes follow a similar maturation route as endosomes, sequentially gaining markers of early endosomal, late endosomal, and lysosomal identity 5,9,10. One of the key questions is how macropinosomes mature immediately after their scission from the plasma membrane and how they gain their endosomal identity. Here, we report a maturation stage of macropinosomes immediately after their scission from the plasma membrane and prior to their acquisition of endocytic markers. Their limiting membrane is densely coated with actin, which is then stripped from the vesicle, allowing the macropinosome to escape the actin cytoskeleton and acquire an endosomal identity. We demonstrate that the PH and FYVE domain-containing protein Phafin2 plays a critical role during this process by a mechanism that involves coincidence detection of phosphoinositide pools and direct control of subcortical actin dynamics.

\section{Results}

Phafin2 shows biphasic localization to macropinosomes. We have previously shown that Phafin2 is required for degradation of endocytosed epidermal growth factor receptors ${ }^{11}$. To further elucidate the mechanism of Phafin2 action on endosomes, we performed live-cell-imaging of Phafin2-GFP in immortalized hTERT-RPE1 retinal pigment epithelial cells (RPE1) stably expressing low levels of the fusion protein. Unexpectedly, we observed distinct subcellular localizations for Phafin2. In addition to the previously described endosomal localization, we observed a striking localization to large vesicles with a clearly defined lumen. Moreover, in cells with a high number of membrane ruffles, we observed bright, short-lived bursts of Phafin2 at vesicles in close proximity to the plasma membrane (Fig. 1a, Supplementary Movie S1). Imaging of Phafin2-GFP together with a plasma membrane marker (MyrPalm-mCherry) ${ }^{12}$ revealed that this transient Phafin2 localization occurred at newly formed macropinosomes immediately after the formation of new vesicles from cup-shaped membrane ruffles (Fig. 1b, c, Supplementary Movie S1).

Tracking of individual vesicles showed two distinct phases of Phafin2 localization to the same macropinosome, a short-lived $(\sim 40 \mathrm{~s})$, transient localization to structures close to the plasma membrane and a second, long-lasting localization to large vacuoles (Fig. 1d, e). The first localization typically occurred in a single pulse, whereas the second localization was characterized by a gradual increase in fluorescence over several minutes. During the first Phafin2 localization, macropinosomes were irregularly shaped and often appeared to be squeezed and subjected to external forces, whereas the second localization occurred on perfectly round macropinosomes (Fig. 1a). While tracking the fate of individual macropinosomes during the first phase of Phafin2 localization, we noted that these were unable to undergo homotypic fusion, even if they were in extensive contact with neighboring vesicles. In comparison, once they reached the second phase of Phafin2 localization, they readily fused if they were in contact with other macropinosomes (Fig. 1f). Taken together, this indicates that the two phases of Phafin2 localization represent two distinct steps of macropinosome maturation.

To test if Phafin2 localization to forming macropinosomes is a general process which occurs in several biological systems, we monitored Phafin2 localization also in HeLa and HT1080 cells transfected with Phafin2-GFP. In both cell types, Phafin2 showed biphasic localization to forming macropinosomes (Supplementary Fig. S1a, b). Many intracellular pathogens exploit macropinosomes to invade host cells or to establish their replicative niche ${ }^{13,14}$. Following Salmonella infection of RPE1 and HeLa cells by time-lapse microscopy, we found that Phafin 2 labeled both Salmonella-induced macropinosomes and Salmonella-containing vacuoles (Fig. 1g, Supplementary Fig. S1c).

Phafin2 labels macropinosomes prior to recruitment of canonical early endocytic markers. To characterize the spatiotemporal localization of Phafin2, we imaged the Phafin2-GFP stable cell line with transiently transfected markers of early endosomes and macropinosomes and assessed their recruitment dynamics relative to the first Phafin2 pulse. The early endocytic adapter protein APPL1 (Fig. 2a, b, Supplementary Movie S2) ${ }^{15}$ localizes to newly formed macropinosomes prior to their acquisition of EEA1 ${ }^{9}$. APPL1 was recruited after the first Phafin2 pulse had occurred, but prior to the second phase of Phafin 2 recruitment. The small GTPases RAB5 (Fig. 2c, d) and RAB31 (Fig. 2e, f) ${ }^{16}$, the RAB5/ PtdIns3P effector Rabankyrin-5 (Fig. $2 \mathrm{~g}, \mathrm{~h}$ ) ${ }^{10}$, and the sorting nexin SNX5 (Fig. 2i, j) also arrived after the first pulse of Phafin2, in parallel with the second gradual localization of Phafin2 to the macropinosome. Snx5 was, in contrast to the other markers, concentrated in subdomains and tubules emanating from macropinosomes. Phafin2 did not localize to clathrin-coated pits by TIRF microscopy, to caveolin-positive structures, or to Endophilin-positive structures (Supplementary Fig. S1d,e,f). These data suggest that Phafin2 is initially also recruited to an immediate-early stage of newly formed macropinosomes that is distinct from and earlier than the better-characterized RAB5positive maturation step (Fig. $2 \mathrm{k}$ ).

Phafin2 localization is dependent on its PH and FYVE domains. To address how these two different maturation steps, nascent and early macropinosomes, are recognized by Phafin2, we generated a series of GFP-tagged truncation mutants lacking different subdomains and assayed their localization (Fig. 3a). The FYVE domain was required for localization of Phafin 2 to both nascent and early macropinosomes. In contrast, the $\mathrm{PH}$ domain was critical for localization to the first phase on macropinosomes, but was not required for localization to the second, endosomal phase (Fig. 3a).

We assessed the lipid-binding specificities of the GST-tagged $\mathrm{PH}$ and FYVE domains using protein-lipid overlay assays. The PH domain bound to PtdIns3P, PtdIns4P, and PtdIns5P (Fig. 3b), with a slight preference for PtdIns3P, whereas the FYVE domain 
a Phafin2-GFP
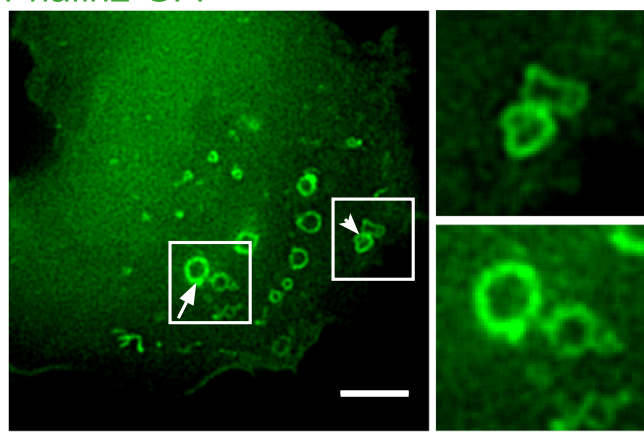

$0 \mathrm{~s}$

C b Phafin2-GFP MyrPalm-mCherry
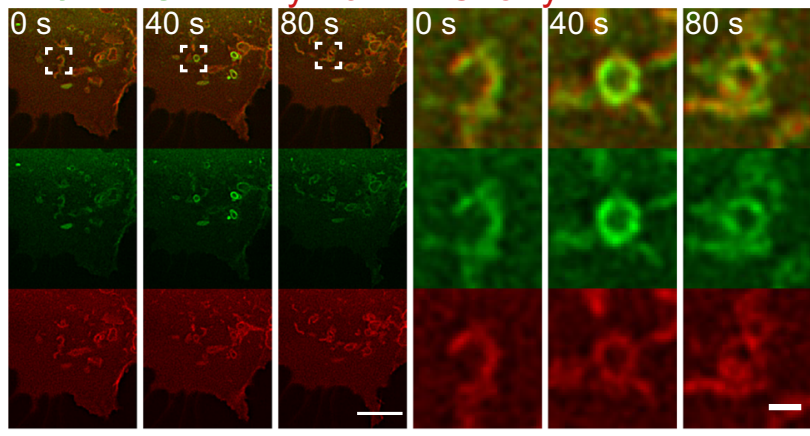

$135 \mathrm{~s}$

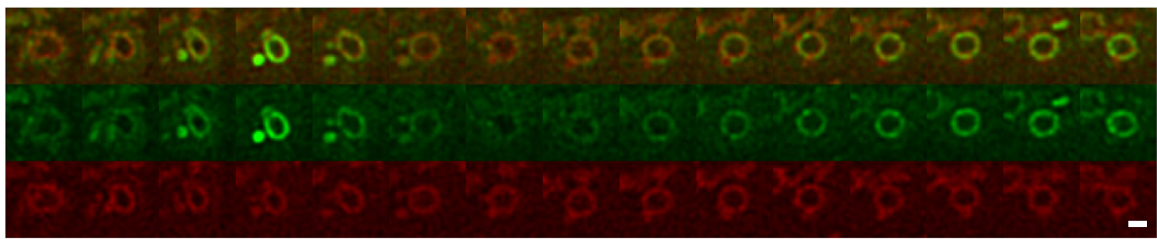

d

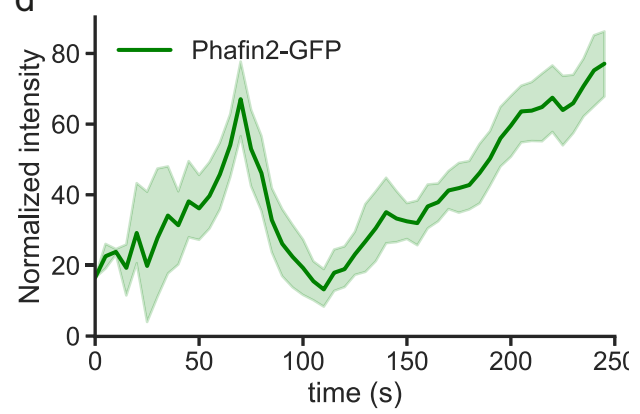

e

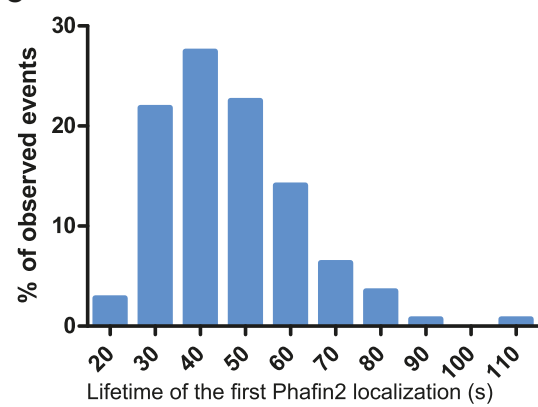

Lifetime of the first Phafin2 localization (s)

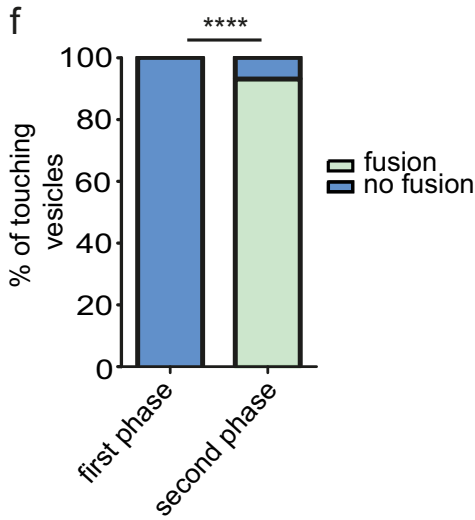

\section{g Phafin2-GFP dsRed-SL}
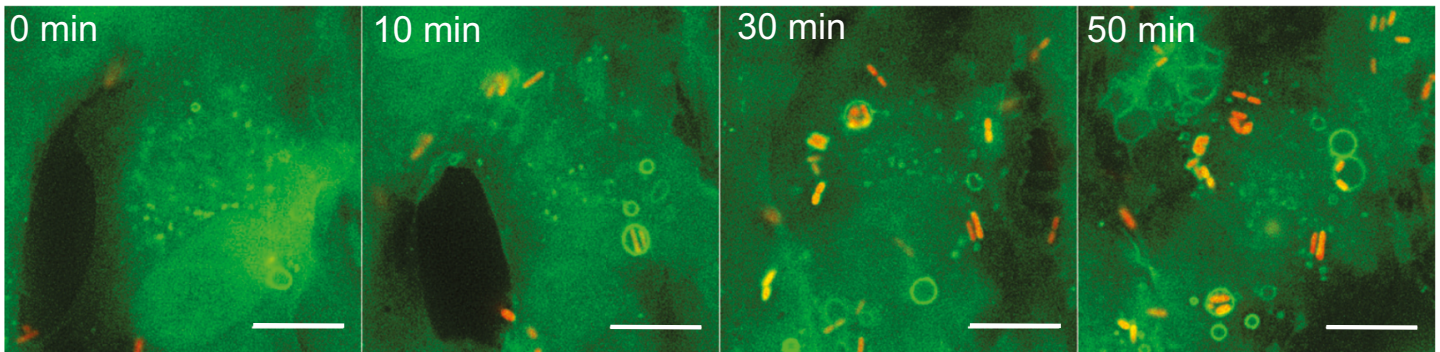

Fig. 1 Phafin2 shows biphasic recruitment to nascent and early macropinosomes. a Micrograph of RPE1 cells expressing Phafin2 showing labeling on two different vesicle classes - round, endosome-like vesicles within the cell (arrow) and non-uniformly shaped structures close to the cell periphery (arrowhead). Scale bar: $10 \mu \mathrm{m}$. Representative image for $n=10$ cells. b Cells co-expressing Phafin2-GFP and MyrPalm-mCherry. Phafin2 transiently localizes to macropinosomes forming from membrane ruffles. Shown are three frames from a time-lapse sequence, spaced $40 \mathrm{~s}$ apart. Representative image for $n=40$ cells. Scale bar: $5 \mu \mathrm{m}$, scale bar inset: $1 \mu \mathrm{m}$. c Sequential images showing Phafin2-GFP dynamics on newly formed macropinosomes; images were acquired every $5 \mathrm{~s}$. Representative image for $n=25$ macropinosomes. Scale bar: $1 \mu \mathrm{m}$. $\mathbf{d}$ Tracking of individual macropinosomes shows a biphasic Phafin2 localization to macropinosomes. All tracks were temporally aligned to the first peak of Phafin2 fluorescence. $n=25$ macropinosomes, mean $+95 \% \mathrm{Cl}$ e Histogram showing the average life-time of the first Phafin2 localization. $n=142$ macropinosomes. $\mathbf{f}$ Macropinosomes labeled with the first Phafin2 localization are fusion incompetent, whereas vesicles readily undergo fusion during the second phase of localization. $n=53$ macropinosomes. Two-sided Fisher's exact test, $p<0.0001$. g Phafin2 localizes to Salmonella-induced macropinosomes and Salmonella-containing vacuoles in RPE1 cells. Representative image for 30 cells. Scale bar: $10 \mu \mathrm{m}$. Statistics source data for $\mathbf{d}$, e, and $\mathbf{f}$ are provided in this paper.

was highly selective for PtdIns3 $\mathrm{P}^{17}$ (Fig. 3b). We did not observe binding to any other phosphoinositide species. We generated Phafin2 constructs bearing point mutations that abolish lipidbinding activity in the $\mathrm{PH}(\mathrm{R} 53 \mathrm{C})^{18}$ and FYVE $(\mathrm{R} 176 \mathrm{~A})^{19}$ domains and assessed their localization behavior. Phafin2(R53C) was still recruited to endosomes and macropinosomes, but the first transient localization to newly formed macropinosomes (Fig. 3c, d, Supplementary Movie S3) was abolished, while Phafin2(R176A) completely lost all membrane association (Fig. 3e, f, Supplementary Movie S4). Thus, PtdIns3P binding by the 

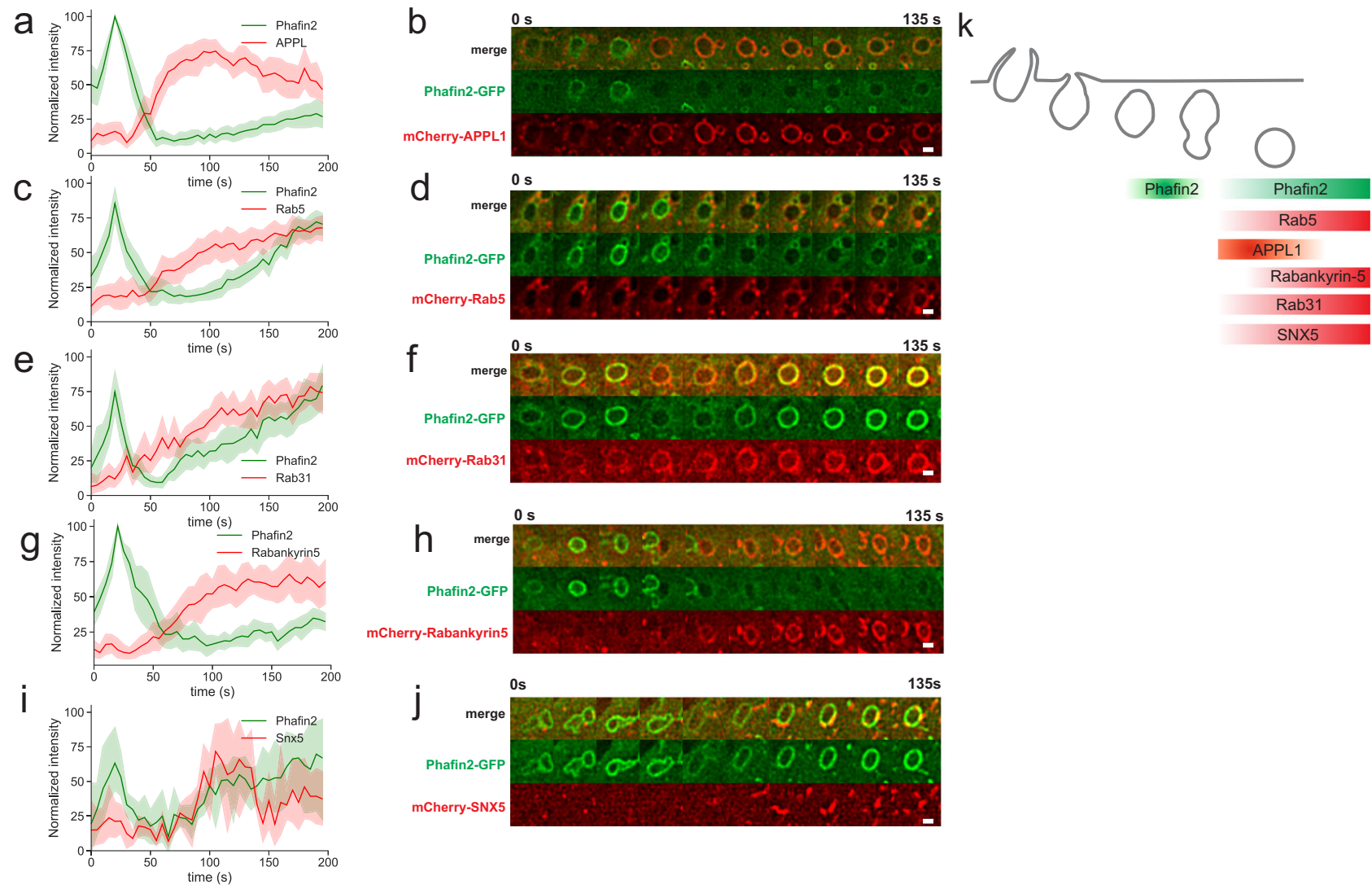

Fig. 2 Mapping Phafin2 dynamics in the endocytic pathway. a Phafin2 is recruited to forming macropinosomes prior to APPL1. Forming macropinosomes acquire a brief burst of Phafin2. Following this burst, Phafin2 is completely lost from these vesicles, and APPL1 is recruited. During further maturation, APPL1 is gradually replaced by a second, slower recruitment of Phafin2. $n=15$ macropinosomes; mean $+95 \% \mathrm{Cl}$. b Sequential images showing Phafin 2 and APPL1 dynamics on a macropinosome. Representative image for $n=15$ macropinosomes. Scale bar: $1 \mu \mathrm{m}$. c Forming macropinosomes acquire a burst of Phafin2, followed by recruitment of RAB5. $n=14$ macropinosomes, mean $+95 \% \mathrm{Cl}$. Phafin2 shows a second gradual recruitment. d Sequential images showing Phafin2 and RAB5 dynamics on a macropinosome. Representative image for $n=14$ macropinosomes. Scale bar: $1 \mu \mathrm{m}$. e RAB31 is recruited after the initial Phafin2 recruitment. $n=10$ macropinosomes, mean $+95 \% \mathrm{Cl}$. f Sequential images showing Phafin2 and RAB31 dynamics on a macropinosome. Representative image for $n=10$ macropinosomes. Scale bar: $1 \mu \mathrm{m}$. g Forming macropinosomes acquire a burst of Phafin2, followed by recruitment of Rabankyrin-5. $n=12$ macropinosomes, mean $+95 \% \mathrm{Cl}$. Macropinosome maturation is accompanied by a second, gradual recruitment of Phafin2. h Sequential images showing Phafin2 and Rabankyrin- 5 dynamics on a macropinosome. Representative image for $n=12$ macropinosomes. Scale bar: $1 \mu$ m. i SNX5 is recruited after the initial Phafin2 recruitment. $n=4$ macropinosomes, mean $+95 \% \mathrm{Cl}$. j Sequential images showing Phafin2 and SNX5 dynamics on a macropinosome. Note that SNX5 localizes to tubules. Representative image for $n=4$ macropinosomes. Scale bar: $1 \mu \mathrm{m}$. $\mathbf{k}$ Schematic overview of the observed recruitment dynamics. Statistics source data for $\mathbf{a}, \mathbf{c}, \mathbf{e}, \mathbf{g}, \mathbf{i}$, and $\mathbf{k}$ are provided in this paper.

FYVE domain of Phafin2 is needed for general membrane association, while the first transient localization of Phafin2 also requires lipid-binding by the $\mathrm{PH}$ domain.

Surprisingly, despite the selectivity of the Phafin2 FYVE domain for PtdIns3P and strict requirement of its lipid-binding activity for membrane association, we only observed minimal colocalization with an HRS-derived 2xFYVE probe for PtdIns3P20,21 (Fig. 3g, h) during the initial Phafin2 pulse. In contrast, $2 x F Y V E$ co-localized strongly during the second phase of Phafin2 localization, suggesting that the second wave of Phafin2 localizes to maturing macropinosomes where PtdIns3P is abundant. Both PtdIns $(3,4,5) \mathrm{P}_{3}$ and PtdIns $(3,4) \mathrm{P}_{2}$ were present at newly formed macropinosomes together with Phafin2 (Fig. 3i-k) and rapidly depleted during the first Phafin2 localization, indicating that lipid substrate for the production of PtdIns3P by this cascade was present.

Early and late Phafin2 recruitments require PtdIns3P generated by different mechanisms. PtdIns3P can be generated by phosphorylation of PtdIns by class II or III PI3Ks, or by dephosphorylation of 3-phosphorylated phosphoinositide species $^{7}$. To disentangle the contribution of different pathways, we blocked the synthesis of PtdIns3P using SAR-405, an inhibitor of the class III PI3K, VPS34 ${ }^{22}$ (Fig. 3m). Treatment with SAR-405 led to a complete displacement of Phafin2 from the endosomal stage (Fig. 3m, Supplementary Movie S5), but preserved the first Phafin2 localization, indicating that the localization of Phafin2 to nascent macropinosomes is VPS34-independent.

To indiscriminately deplete PtdIns3P from all cellular membranes, we overexpressed the PtdIns3P phosphatase MTM1 or a catalytically inactive mutant, MTM1(C375S) as control (Fig. 3n) ${ }^{23}$. Overexpression of MTM1 displaced Phafin2 from both the first and second localization stage, whereas expression of MTM1(C375S) did not affect Phafin2 localization. Likewise, acute release of mitochondrially tethered MTM1 using a reversible dimerization system ${ }^{24}$ resulted in depletion of Phafin2 from forming macropinosomes (Supplementary Movie S6), whereas release of MTM1(C375S) did not affect Phafin2 localization (Supplementary Movie S7). These results suggest that Phafin2 recognizes a VPS34-independent PtdIns3P pool at newly formed macropinosomes and a VPS34-derived endosomal pool at maturing macropinosomes for its biphasic recruitment. 
a

\begin{tabular}{|c|c|c|c|c|}
\hline & & Mutant & $\begin{array}{c}\text { Forming } \\
\text { macropinosome }\end{array}$ & Endosome \\
\hline \begin{tabular}{|l|l|}
$\mathrm{N}$ & $\mathrm{PH}$ \\
\end{tabular} & 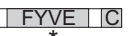 & WT & + & + \\
\hline $\mathrm{N} \| \mathrm{PH}$ & $\left|F Y^{*} E\right| C \mid C$ & $\mathrm{R} 176 \mathrm{~A}$ & - & - \\
\hline $\mathbb{N} \| \stackrel{*}{*}{ }^{*}$ & \begin{tabular}{|l|l|} 
FYVE & $\mathrm{C}$ \\
\end{tabular} & R53C & - & + \\
\hline$\Delta \square \mathrm{PH}$ & \begin{tabular}{l|l} 
FYVE & $\mathrm{C}$ \\
\end{tabular} & $\Delta \mathrm{NT}$ & + & + \\
\hline $\mathbb{N} \quad \Delta$ & \begin{tabular}{||l|l|} 
FYVE & $\mid C$ \\
\end{tabular} & $\Delta \mathrm{PH}$ & - & + \\
\hline \begin{tabular}{|l|l}
$\mathrm{N}$ & $\mathrm{PH}$
\end{tabular} & $\Delta \quad \mathrm{C}$ & $\Delta F Y V E$ & - & - \\
\hline \begin{tabular}{|l|l}
$\mathrm{N}$ & $\mathrm{PH}$
\end{tabular} & $\mid$ FYVE $\mid \Delta \Delta$ & $\Delta \mathrm{CT}$ & + & + \\
\hline
\end{tabular}

b

Ptdlns3P

Ptdlns4P

Ptdlns5P

Ptdlns3,4P2

Ptdlns3,5P2

Ptdlns4,5P2

Ptdlns3,4,5P3
C

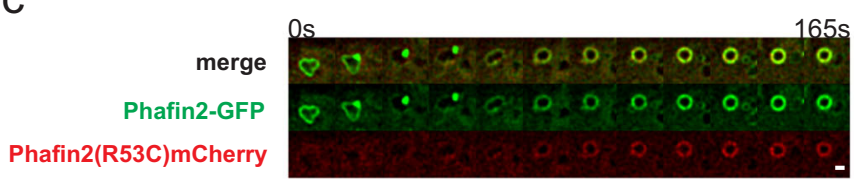

e

e merge

Phafin2-GFP

Phafin2(R176A)mCherry

0s

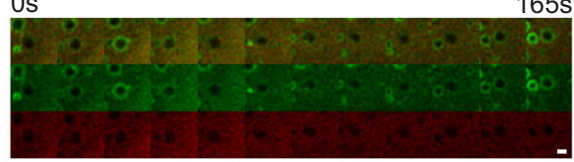

g

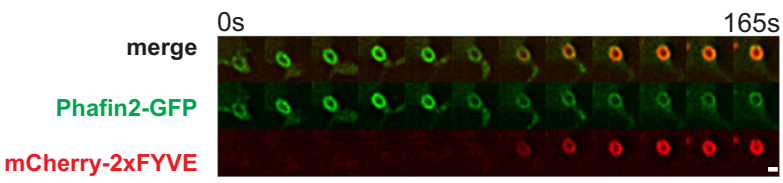

i

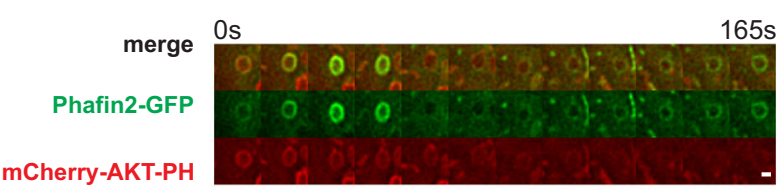

k

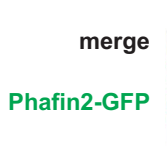

mTagRFP-TAPP-2xPH

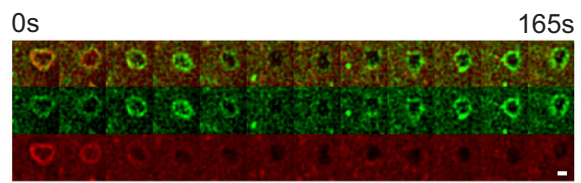

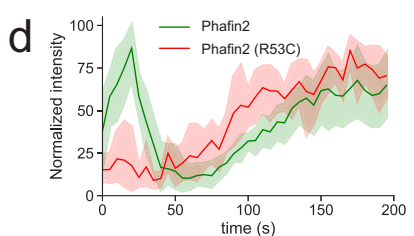

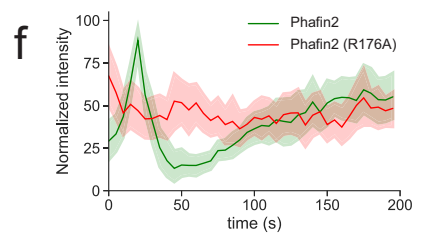

$\mathrm{h}$
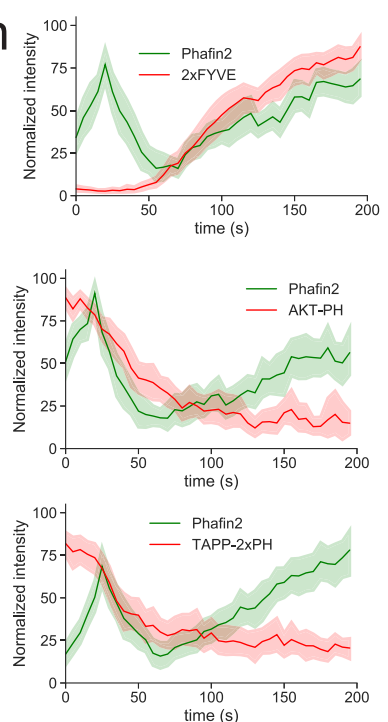

n Phafin2 not localized

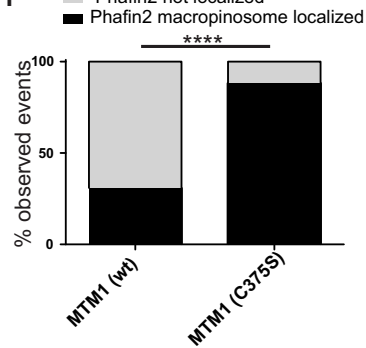

m

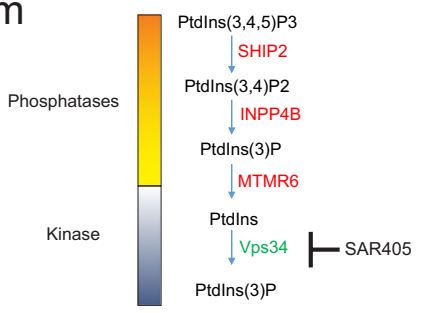

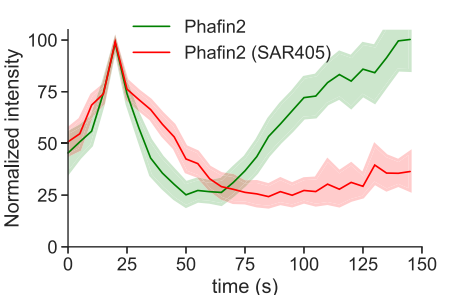

GST-FYVE

Fig. 3 Mutational analysis of Phafin2. a Mutational analysis of Phafin2. GFP fusions of Phafin2 truncation and point mutants were tested for their subcellular localization. b Protein-lipid binding assay of Phafin2 PH and FYVE domains. c Sequential images of a macropinosome showing recruitment dynamics of Phafin2 (WT) and Phafin2 with a defective PH domain (Phafin2(R53C)). Representative image for $n=6$ macropinosomes. Scale bar: $1 \mu$ m. d Localization dynamics of Phafin2 and Phafin2 with a defective PH domain (R53C). $n=6$ macropinosomes, mean $+95 \% \mathrm{Cl}$. Phafin2 R53C is unable to localize to newly formed macropinosomes, whereas the second, endosomal localization is unaffected. e Sequential images of a macropinosome showing recruitment dynamics of Phafin2 (WT) and Phafin2 with a defective FYVE domain (Phafin2 (R176A)). Representative image for $n=15$ macropinosomes. Scale bar: $1 \mu \mathrm{m}$. $\mathbf{f}$ Localization dynamics of Phafin2 and Phafin2 with a defective FYVE domain (R176A). $n=15$ macropinosomes, mean $+95 \% \mathrm{Cl}$. Mutation of the FYVE domain abolishes all membrane localization. $\mathbf{g}$ Sequential images showing Phafin2 and the Ptdlns3P probe mCherry-2xFYVE on a macropinosome. Representative image for $n=20$ macropinosomes. Scale bar: $1 \mu \mathrm{m}$. $\mathbf{h}$ Localization dynamics of Phafin2 in relation to the Ptdlns3P probe mCherry-2xFYVE. $n=20$ macropinosomes, mean $+95 \% \mathrm{CI}$. i Sequential images showing Phafin2 and the Ptdlns $(3,4,5) \mathrm{P}_{3}$ probe AKT-PH on a macropinosome. Representative image for $n=19$ macropinosomes. Scale bar: $1 \mu \mathrm{m}$. j Localization dynamics of Phafin2 in relation to the Ptdlns $(3,4,5) P_{3}$ probe AKT-PH. $n=19$ macropinosomes, mean $+95 \%$ Cl. $\mathbf{k}$ Sequential images showing Phafin2 and the Ptdlns $(3,4) \mathrm{P}_{2}$ probe TAPP-2xPH on a macropinosome. Representative image for $n=20$ macropinosomes. Scale bar: $1 \mu \mathrm{m}$. I Localization dynamics of Phafin2 in relation to the Ptdlns $(3,4) P_{2}$ probe TAPP-2xPH. $n=20$ macropinosomes, mean $+95 \% \mathrm{Cl}$. $\mathbf{m}$ Treatment of Phafin2-GFP expressing cells with the VPS34 inhibitor SAR-405 does not affect the first localization, but abolishes the second localization phase $(n=51$ macropinosomes each, mean $+95 \% \mathrm{Cl})$. $\mathbf{n}$ Overexpression of the PI3 phosphatase MTM1, but not of the catalytically inactive MTM1 (C375S) displaces Phafin2 from macropinosomes ( $n=75$ (WT) and 69 (C375S) cells). Two-sided Fisher's exact test, $p<0.0001$. Statistics source data for $\mathbf{c}, \mathbf{e}, \mathbf{i}, \mathbf{k}$, and $\mathbf{m}$ are provided in this paper.

macropinosome closure ${ }^{8}$. The first phase of Phafin2 localization displayed remarkably similar spatiotemporal dynamics as SidCP4C, a high-affinity probe for PtdIns4P 26 (Fig. 4c, d). In line with this, a synthetic construct where we replaced the Phafin2 $\mathrm{PH}$ domain with the SidM-P4M PtdIns4P binding domain (SidMFYVE), faithfully recapitulated both the first and second Phafin2 localization (Fig. 4e, f). The same construct with a defective FYVE domain (SidM-FYVE(R176A)) could still localize to nascent macropinosomes, but did not bind to the second phase (Fig. $4 \mathrm{~g}$, $\mathrm{h}$ ). This suggests that forming macropinosomes contain a sizeable pool of PtdIns4P, which could drive the first localization of Phafin2. It is likely that the $\mathrm{PH}$ domain of Phafin2 is only a weak PtdIns4P binder, as an isolated $\mathrm{PH}$ domain or Phafin2 with a defective FYVE domain is unable to localize to any membrane structures. Therefore, we hypothesized that the first localization of Phafin 2 might rely on coincidence sensing of PtdIns3P by the FYVE domain and PtdIns4P by the $\mathrm{PH}$ domain.

To characterize the binding properties of Phafin2 towards membranes of differing phosphoinositide compositions, we performed liposome flotation assays. We used liposomes with an endosome-like lipid composition doped with PtdIns3P (0.5\%), PtdIns4P (5\%), or both (Fig. 4i, j), with PtdIns to balance phospholipid composition. Phafin2 bound to the liposomes containing a single phosphoinositide species but this was strongly enhanced using liposomes containing both PtdIns3P and PtdIns4P. Phafin2(R53C) bound to liposomes with PtdIns3P, and, surprisingly, also bound to PtdIns4P-containing liposomes. However, binding was not enhanced towards liposomes doped with both PtdIns3P and PtdIns4P. Phafin2(R176A) showed strongly reduced binding to PtdIns3P, whereas binding to PtdIns4P was not affected. For this mutant, the enhanced binding to PtdIns3P/PtdIns4P liposomes was impaired (over 3 -fold in wild type vs. $<1.5$-fold for $\mathrm{R} 176 \mathrm{~A})$. These in vitro and in vivo results suggest that minor amounts of PtdIns3P are able to strongly enhance recruitment of Phafin2 to membranes containing PtdIns4P, and that the participation of both the PH and FYVE domains is required.

The C-terminal acidic tail of Phafin2 suppresses spurious membrane recruitment of Phafin2. Apart from the two lipidbinding motifs, Phafin 2 contains a highly acidic and negatively charged C-terminal tail, which was proposed to block the $\mathrm{PH}$ domain $^{27}$. We generated a Phafin2 construct lacking this domain (Phafin $2 \Delta \mathrm{C}$ ), which remained capable of macropinosome localization but also showed an additional localization to the plasma membrane (Fig. 4k). Plasma membrane recruitment of Phafin $2 \Delta \mathrm{C}$ required binding to PtdIns3P, as Phafin $2 \Delta \mathrm{C}$ with a defective FYVE domain (R176A) was cytosolic (Fig. 4k), in line with the inability of an isolated $\mathrm{PH}$ domain to cause membrane association. Phafin $2 \Delta \mathrm{C}$ also required PtdIns4P to localize to the plasma membrane, as inhibition of PI4KIIIa (100 nM GSK-A1), the major source of PtdIns4P at the plasma membrane ${ }^{28}$, effectively removed Phafin $2 \Delta \mathrm{C}$ from the plasma membrane (Fig. 4l, m). Taken together, our data indicate that Phafin2 needs to bind to both PtdIns3P and PtdIns4P for effective membrane recruitment if PtdIns3P is not abundant.

Phafin2 is required for fluid-phase uptake by macropinocytosis. To assess the functional role of Phafin2 in macropinocytosis, we generated RPE1 cells completely lacking Phafin2 (Supplementary Fig. S2). We measured macropinocytosis using flow cytometry and dextran as a fluid-phase marker. WT cells stimulated with HGF showed robust dextran uptake, whereas Phafin KO strongly reduced dextran uptake (Fig. 5a). This effect was especially pronounced in HGF-treated cells, as unstimulated cells formed only a few and small macropinosomes (Supplementary Fig. S3a, b, c). Depletion of Phafin2 with two different siRNAs resulted in a strong reduction in fluid-phase uptake (Supplementary Fig. S3e, S3f). Phafin2 KO cells also internalized less dextran in a similar microscopy-based assay (Supplementary Fig. 3d). We conclude that Phafin2 is critical for efficient macropinocytosis.

We next used the Phafin2 - / cell line to follow macropinocytosis by live-cell imaging. Cells were transfected with GFP$2 \mathrm{xFYVE}$ to label macropinosomes at the endosomal stage, and with Lifeact-SNAP to label membrane ruffles. In WT cells, we readily observed the formation of multiple large vesicles (Fig. 5b, c, Supplementary Movie S9). In contrast, despite heavy ruffling in Phafin $2 \mathrm{KO}$ cells, we only occasionally observed the formation of large vesicles by macropinocytosis (Fig. 5b, c, Supplementary Movie S9).

To monitor the fate of macropinosomes from their formation at the plasma membrane to endosomal stages, we used WT and Phafin2 - / - cell lines stably expressing mNeonGreen(mNG) $-2 x F Y V E$ and MyrPalm-mCherry and studied these by live imaging and automated vesicle tracking. Tracking of mNG$2 x F Y V E$ labeled macropinosomes $(>1 \mu \mathrm{m}$ diameter) showed that macropinosomes in Phafin2 $-/-$ cells were much smaller than in WT cells (Fig. 5d). Moreover, knockout cells completely lacked large macropinosomes $\left(>10 \mu \mathrm{m}^{2}\right)$ (Fig. 5e). We observed similar effects when we depleted Phafin2 using siRNA. Using Rabankyrin-5 as a macropinosome marker, we noted that cells depleted for Phafin2 showed significantly fewer macropinosomes than control cells, whereas stable expression of siRNA-resistant 
a
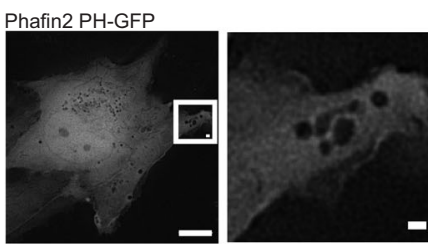

b Phafin2-GFP

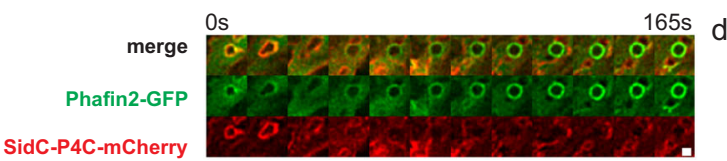

e



mCherry-2XFYVE

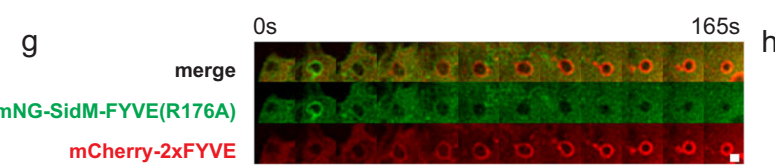

mCherry-2xFYVE
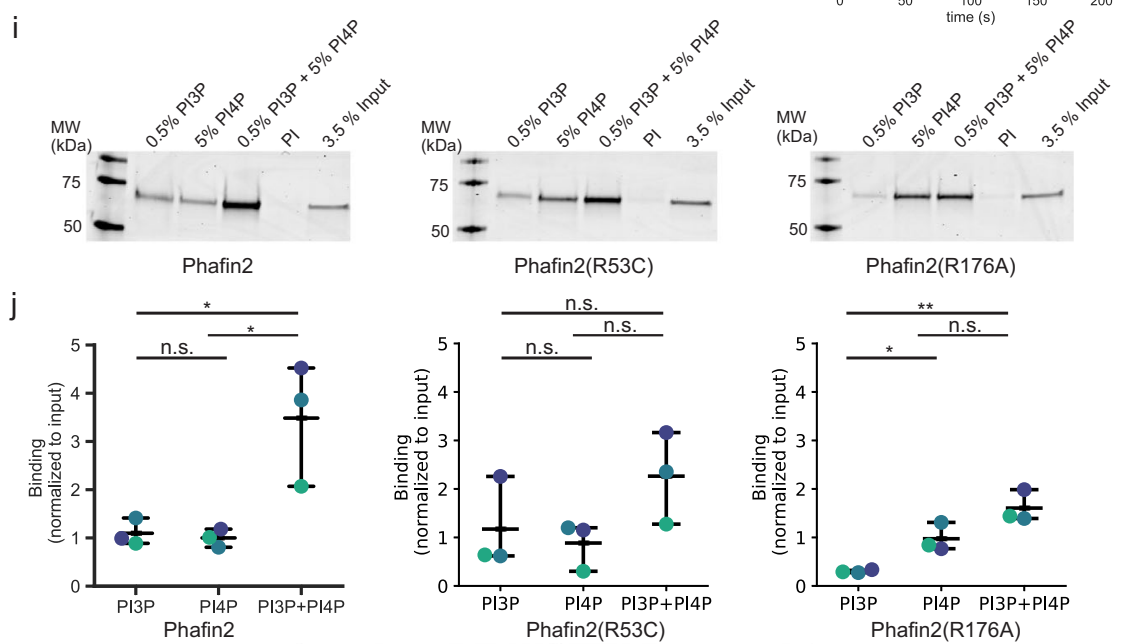

$\mathrm{k}$
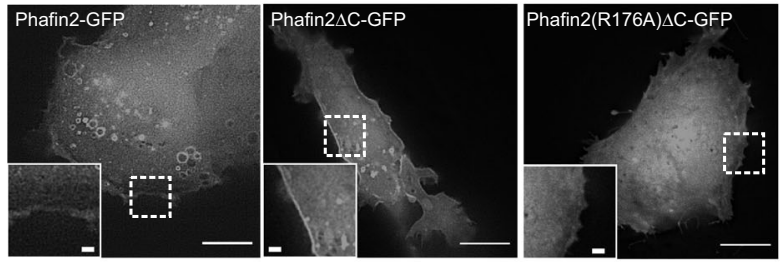

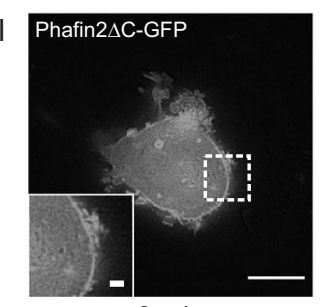

$0 \mathrm{~min}$

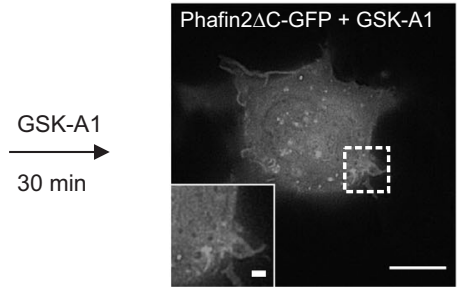

$30 \mathrm{~min}$

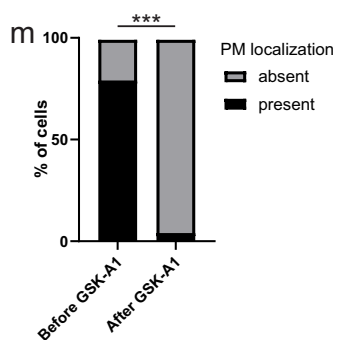

Phafin2 was able to rescue this defect (Supplementary Fig. S3f-i). These experiments indicate that Phafin2 is required for the formation of normal macropinosomes.

Phafin2 is required for early steps of macropinosome formation. Phafin $2-/-$ cells showed robust membrane ruffling, suggesting that these cells should be able to initiate macropinosomes. To address why macropinocytosis was nevertheless inhibited in Phafin $2-/-$ cells, we tracked the fate of individual macropinosome cups using MyrPalm-mCherry to visualize forming macropinosomes and mNeonGreen-tagged 2xFYVE (mNG-2xFYVE) to indicate the establishment of endosomal identity. In WT cells, $\sim 40 \%$ of macropinosomes successfully 
Fig. 4 Two independent Ptdlns3P pools control the biphasic recruitment of Phafin2. a An isolated Phafin2 PH domain does not show membrane localization. Representative image for $n=12$ cells. Scale bar: $10 \mu \mathrm{m}$. b A dual Phafin2 PH domain (2xPH) localizes to the plasma membrane and to forming macropinosomes but not to later, endosomal stages. Representative image for $n=19$ cells. Scale bar: $10 \mu$ m. c Sequential images showing Phafin 2 and the Ptdlns4P probe SidC-P4C on forming macropinosomes. Representative image for $n=25$ macropinosomes. Scale bar: $1 \mu \mathrm{m}$. d Localization dynamics of Phafin2 in relation to the Ptdlns4P probe SidC-P4C. $n=25$ macropinosomes, mean $+95 \% \mathrm{Cl}$. e Sequential images showing the synthetic coincidence sensor SidM-FYVE(Phafin2) and the Ptdlns3P probe 2xFYVE on forming macropinosomes. Representative image for $n=25$ macropinosomes. Scale bar: $1 \mu \mathrm{m}$. $\mathbf{f}$ Localization dynamics of SidM-FYVE(Phafin2) in relation to the Ptdlns3P probe $2 x \mathrm{FYVE} . n=25$ macropinosomes, mean $+95 \% \mathrm{Cl}$. $\mathbf{g}$ Sequential images showing the synthetic coincidence sensor SidM-FYVE ${ }^{(\text {Phafin2) }}(\mathrm{R} 176 \mathrm{~A})$ and the Ptdlns3P probe 2xFYVE on forming macropinosomes.

Representative image for $n=25$ macropinosomes. Scale bar: $1 \mu \mathrm{m}$. h Localization dynamics of SidM-FYVE(Phafin2) (R176A) in relation to the Ptdlns3P probe 2xFYVE. $n=25$ macropinosomes, mean $+95 \%$ Cl. i Liposome flotation assays showing Phafin2, Phafin2(R53C) and Phafin2(R176A) binding to liposomes. Shown is the liposome fraction. Representative gel from $n=3$ experiments. $\mathbf{j}$ Quantification of Phafin2, Phafin2(R53C) and Phafin2(R176A) binding to liposomes. $n=3$ experiments, mean $+95 \%$ Cl. One-way ANOVA with Tukey's post-test. $p=0,0114$ (WT), n.s. (R53C) and $p=0.0024$ (R176A). ${ }^{\star} p<0.05,{ }^{\star \star} p<0.01$, n.s. not statistically significant. k Exemplary image showing the localization of Phafin2-GFP, Phafin2 $\Delta C, P h a f i n 2 \Delta C(R 176 A)$.

Representative image for 20 cells each. Scale bar: $10 \mu \mathrm{m}$. I Treatment of cells expressing Phafin $2 \Delta \mathrm{C}$ before and after treatment with the PI4K inhibitor GSKA1. Representative image for 81 cells. Scale bar: $10 \mu \mathrm{m}$. $\mathbf{m}$ Quantification of (I). Two-sided Fisher's exact test, $n=81$ cells, $p=0.0001$. Statistics source data and detailed statistics for $\mathbf{d}, \mathbf{f}, \mathbf{h}, \mathbf{j}$ and $\mathbf{m}$ are provided in this paper.

gained endosomal identity (Fig. 5f, g, h, i, Supplementary Movie S10). In contrast, in Phafin2 - /- cells, only $20 \%$ of forming macropinosomes successfully gained endosomal identity, whereas the rest collapsed and receded to the plasma membrane before mNG-2xFYVE was recruited (Fig. 5f, Supplementary Movie S11). The establishment of endosomal identity, defined by mNG-2xFYVE, was a strong indicator of successful macropinocytosis. The collapse of macropinosomes invariably happened prior to $\mathrm{mNG}-2 \mathrm{xFYVE}$ recruitment, whereas virtually all vesicles that gained mNG-2xFYVE were stable and matured. This suggests that Phafin2 acts at the transition between cup-shaped membrane ruffles and the establishment of endosomal identity.

We observed in rare cases that receding macropinocytic cups appeared to form tubules, which could indicate that they were still connected or had reconnected to the plasma membrane (Fig. 5j, Supplementary Movie S12). To pinpoint the arrival of Phafin2 in relation to sealing of the macropinosome, we performed a $\mathrm{pH}$ based scission assay using a surface-displayed $\mathrm{pH}$-sensitive red fluorescent protein, Phuji ${ }^{29,30}$ (Fig. 5k). Cells were perfused with imaging solutions buffered to $\mathrm{pH} 5.5$ and $\mathrm{pH} 7.5$, alternating every 5 seconds. This caused cycles of quenching and unquenching of Phuji fluorescence on surface-exposed structures but not on sealed vesicles (Supplementary Movie S13; Supplementary Fig. 4a). By measuring Phafin2 intensity and the time point of sealing, we found that sealing directly preceded the recruitment of Phafin2 (Fig. 5l). An analogous assay using the calcium-sensitive protein GCAMP6, which was quenched using EGTA and unquenched by a calcium-containing buffer, also showed that Phafin2 arrived directly after sealing of the vesicle (Supplementary Fig. 4b). This suggests that Phafin 2 is not directly involved in the scission of the macropinosome from the plasma membrane, as it is only found on sealed vesicles. Taken together, our findings indicate that Phafin2 is critical for successful macropinosome formation and that it is involved in the step between the closure of the macropinocytic cup and the establishment of endosomal identity.

The first phase of Phafin2 localization coincides with actin rearrangements around macropinosomes. The formation of macropinosomes is tightly linked to actin dynamics ${ }^{1}$. In order to visualize the formation of Phafin2-labeled macropinosomes in relation to actin-driven membrane ruffles, we used highresolution oblique plane light sheet microscopy (Fig. 6a, Movie S14). We found that the first phase of Phafin2 localization coincides with the major restructuring of the actin cytoskeleton. Actin-coated macropinosomes emerged from the base of membrane ruffles, gained Phafin2, and lost their actin coat. Highresolution live-cell imaging of Phafin2 and the actin marker Lifeact-mCherry showed that newly formed Phafin2-labeled macropinosomes were coated with a dense network of actin filaments (Fig. 6b, c, Supplementary Movie S15). We observed a transient peak of Lifeact-mCherry localization to newly formed macropinosomes, which coincided with Phafin2 recruitment. Actin-associated proteins, such as the actin crosslinking protein FilaminA and the myosin motor protein Myo1E, were present during the first phase of Phafin2 localization. Myo1E-which localizes to macropinosome cups and has been shown to drive phagocytic cup constriction ${ }^{31-33}$, localized directly prior to Phafin2 recruitment (Fig. 6d, e). FilaminA co-localized with Phafin2 on forming macropinosomes and dissociated together with actin (Fig. 6f, g). This indicates that during the first Phafin2 localization, macropinosomes are coated in a dense, highly crosslinked actin network.

Live-cell imaging of Phafin2 and either Lifeact or FilaminA showed that Phafin2 localization of macropinosomes coincided with the removal of this actin network from the macropinosome. During this "uncoating" process, macropinosomes were subject to squeezing forces that pushed them into the inner parts of the cell (Fig. 6h, i, Supplementary Movies S16 and S17). Moreover, we observed gaps in the actin cytoskeleton lining the macropinosomes by both live-cell and 3D SIM imaging (Fig. 6h,i, j). These gaps were visible in both FilaminA and Lifeact expressing cells and coincided with the first Phafin2 localization. Phafin2-positive vesicles were squeezed through these gaps, which allowed the vesicles to leave their actin coat behind and acquire a completely round-vesicle-like shape (Supplementary Movie S16). Directly after vesicles were forced through this gap, they lost the first Phafin2 localization and gained endosomal identity (Fig. 6k).

Interestingly, we observed that macropinosomes in cells lacking Phafin 2 retained FilaminA around the limiting membrane until they ultimately collapsed (Supplementary Fig. S5a, b, c, Supplementary Movie S18), suggesting that in Phafin2 KO cells, macropinosomes are enclosed in a densely crosslinked actin coat. Depleting FilaminA suppressed the Phafin2 KO phenotype and allowed formation of large macropinosomes in Phafin2 KO cells (Supplementary Fig. S5d, e), suggesting that the Phafin2 KO phenotype might be caused by a failure to remove the actin coat surrounding the macropinosome, which can be remedied by reducing actin crosslinking. These observations pose the question of how Phafin 2 and actin act during macropinosome formation. We observed that cells depleted for Phafin2 by siRNA showed a strongly increased number of membrane ruffles, whereas the introduction of a siRNA-resistant allele reduced this effect 
a

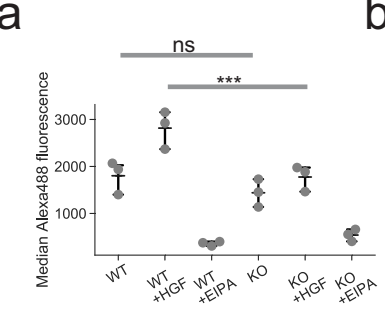

b

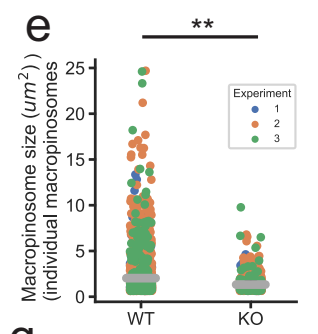

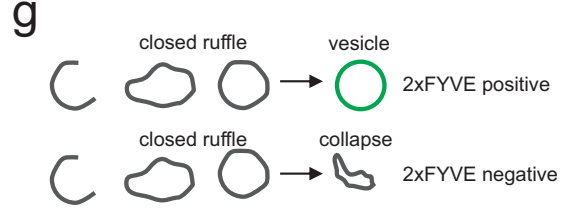

$h_{\text {mNG-2xFYVE MyrPalm-mCh }}^{\text {Successful vesicle formation (WT) }}$

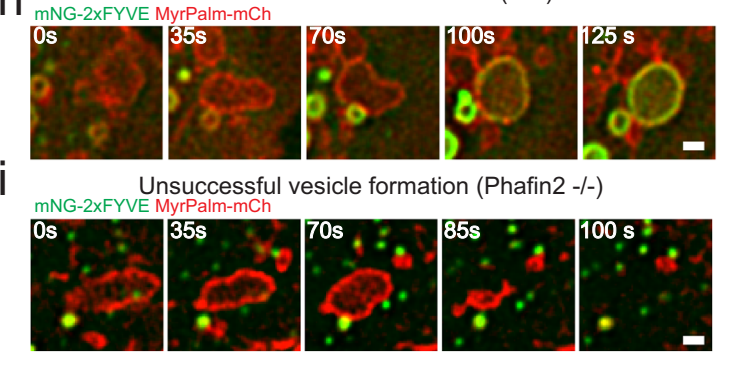

C
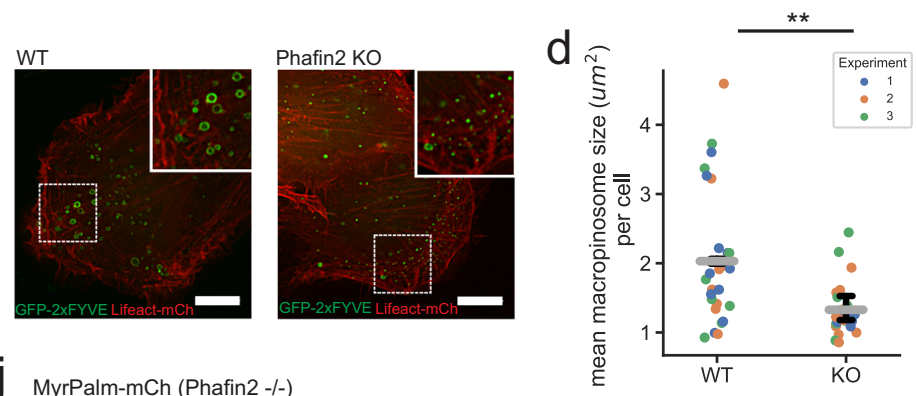

j MyrPalm-mCh (Phafin2 -l-)

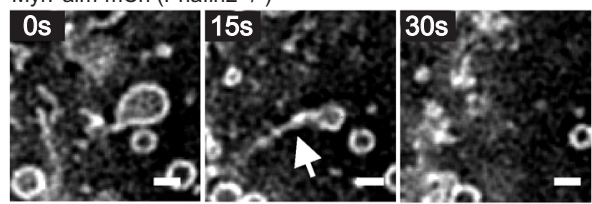

$\mathrm{k}$

$\mathrm{pH} 5$
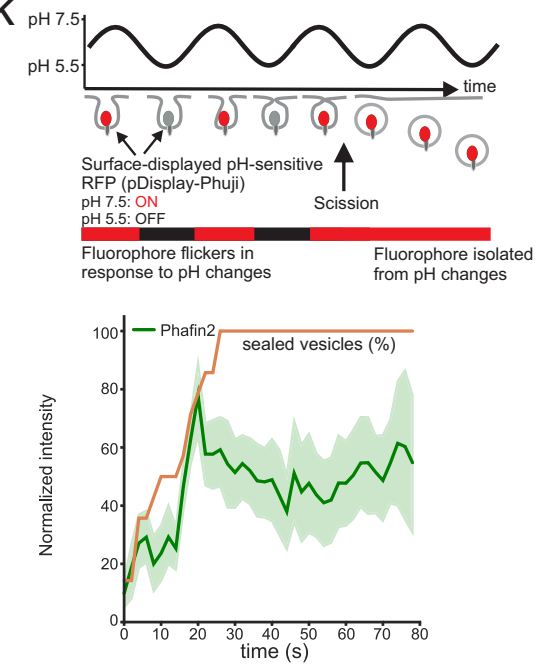

Fig. 5 Phafin2 is required for efficient macropinocytosis. a Flow cytometry measurement of dextran uptake (10 kDa, Alexa488) in WT and Phafin2 knockout cells. Phafin2 knockout cells show reduced dextran uptake under stimulated conditions. $n=3$ experiments, mean $+95 \% \mathrm{Cl}$, ANOVA with Tukey's post-test, $p<0.0001$. ${ }^{\star \star} p<0.001$, n.s. not statistically significant. b Formation of new macropinosomes ( $>1$ um in diameter) in Phafin2 WT and $\mathrm{KO}$ cells. Macropinosome formation was scored over 20 min of imaging time. $n=14$ (wt) and 8 (KO) cells, mean $+95 \% \mathrm{Cl}$, two-sided t-test, $p=0.043$. ${ }^{\star} p<0.05$. c Exemplary image showing macropinocytosis in WT and Phafin2 knockout cells (single frame extracted from the movies analyzed in (d)). Scale bar: $10 \mu \mathrm{m}$. d Phafin2 knockout cells have smaller macropinosomes than WT cells. Plotted are the mean macropinosome size $\left(>0.64 \mu m^{2}\right.$ area) per individual cell and the mean per experiment $+95 \% \mathrm{Cl} . n=3$ experiments, two-sided t-test, $p=0.0026$. e Phafin2 knockout cells are unable to form large macropinosomes. Plotted is the size of individual macropinosomes ( $>0.64 \mu \mathrm{m}^{2}$ area) and the mean per experiment $+95 \% \mathrm{Cl}$. $n=3 \mathrm{experiments,} \mathrm{two-}$ sided t-test, $p=0.0076$. f Phafin2 knockout cells are less successful in forming macropinosomes. Plotted is the success rate per individual cell and the mean per experiment $+95 \% \mathrm{Cl} . n=3$ experiments, two-sided t-test, $\mathrm{p}=0.0038$. $\mathbf{g}$ Schematic showing the phenotypes scored in $\mathbf{f}$. $\mathbf{h}$ Sequential images showing successful macropinosome formation in WT cells. Scale bar: $1 \mu \mathrm{m}$. Representative images for $n=25$ cells. i Sequential images showing collapsing macropinosomes in Phafin2 knockout cells. Scale bar: $1 \mu \mathrm{m}$ Representative images for $n=24$ cells. j Sequential images showing macropinosome-associated tubules during collapse of a macropinosome. Scale bar: $1 \mu \mathrm{m}$ Representative image for 15 cells. $\mathbf{k}$ Assay measuring the closure of macropinosomes. Cells displaying pH-sensitive pHuji-RFP on their surface were alternatingly perfused with buffers at pH 5.5 and 7.5, and localization of Phafin2 was evaluated in comparison to pHuji. I Quantification of Phafin2 localization to forming macropinosomes in relation to vesicle sealing. $n=15$ macropinosomes,

mean $+95 \% \mathrm{Cl}$. Statistics source data and detailed statistics for $\mathbf{a}, \mathbf{b}, \mathbf{d}-\mathbf{f}$, and $\mathbf{I}$ are provided in this paper.

(Fig. 7a, b). This led us to ask if Phafin2 could influence actin dynamics at forming macropinosomes.

The Phafin2 PH domain binds directly to F-actin. Some PH domains, such as the $\mathrm{PH}$ domain of $\mathrm{BTK}^{34}$, can directly bind to F-actin. Based on the close interaction of Phafin 2 and actin, we therefore tested if Phafin2 can directly bind to F-actin via its $\mathrm{PH}$ domain. We performed in vitro F-actin binding assays-using recombinant $\mathrm{His}_{6}$-MBP-fused Phafin2, $\mathrm{His}_{6}-\mathrm{MBP}-\mathrm{PH}$, and $\mathrm{His}_{6}{ }^{-}$ MBP-FYVE domains, as well as His6-MBP as a control-and measured the F-actin-bound fraction of Phafin2. Both full-length Phafin2 and the Phafin2 $\mathrm{PH}$ domain showed robust binding to filamentous actin, whereas the Phafin2 FYVE domain did not show binding above the background (Fig. 7c, d). This indicates that Phafin 2 can bind directly to F-actin and that this binding is mediated by the $\mathrm{PH}$ domain.

Phafin2 modulates actin cytoskeleton dynamics. Phafin2 is transiently recruited to forming macropinosomes, and the recruitment coincides with the uncoating of the macropinosome from actin. It is, therefore, tempting to speculate that Phafin2 could locally modulate actin dynamics in close proximity to the plasma membrane. We therefore tested if membrane-associated Phafin 2 could modulate actin dynamics. For this, we exploited our observation that Phafin2 lacking the C-terminus (Phafin2 $\Delta \mathrm{C}$ ) localized not only to forming macropinosomes but also to the 
a Phafin2-GFP Lifeact-SNAP

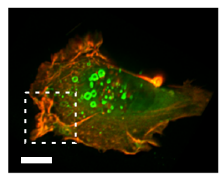

b
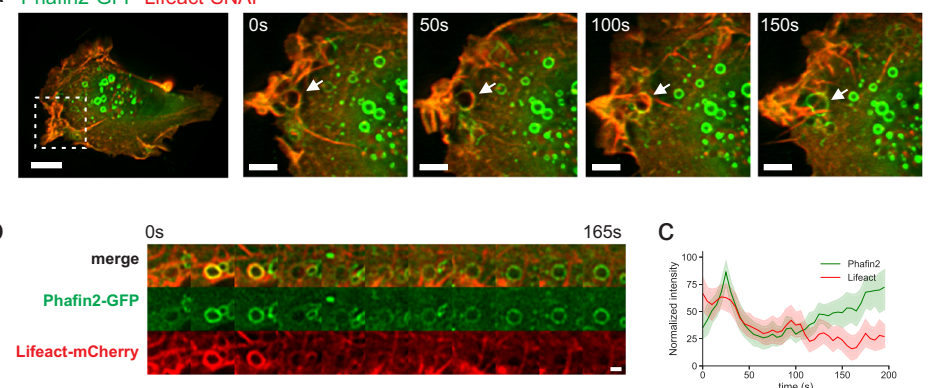

d

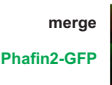

MAPPLE-Myo1E

f

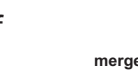

nesere

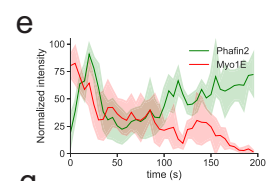

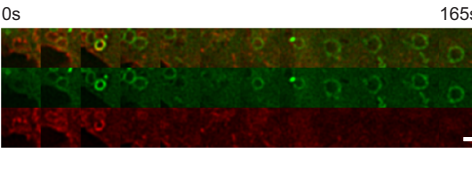

$165 \mathrm{~s}$

$\mathrm{g}$

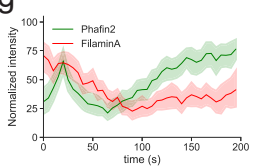

$\mathrm{h}$ merge
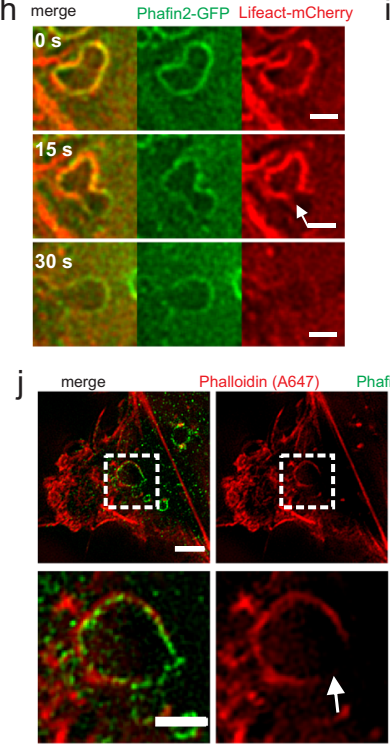
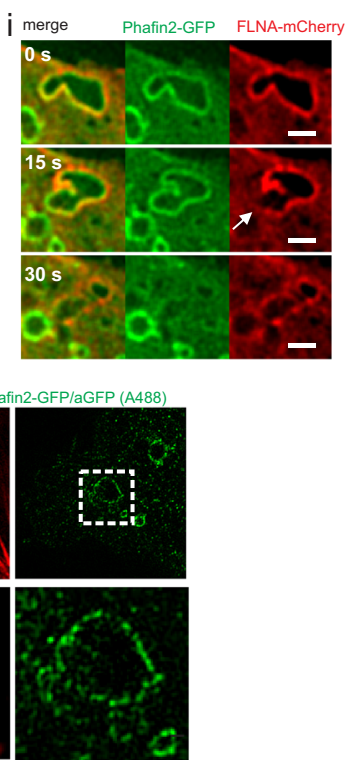

$\mathrm{k}$

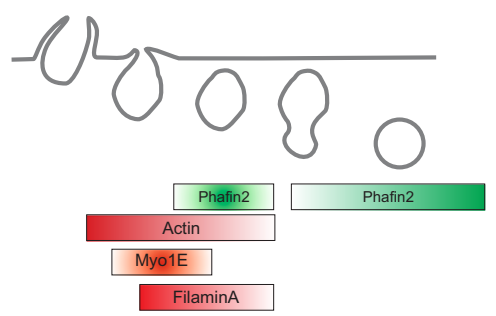

Fig. 6 Phafin2 localization coincides with restructuring of the actin cytoskeleton. a Light sheet imaging of RPE1 cells expressing Phafin2-GFP and LifeactSNAP. Representative image of $n=5$ cells. Scale bar: $10 \mu \mathrm{m}$, inset: $5 \mu \mathrm{m}$. b Sequential images showing Phafin 2 and Lifeact on macropinosomes. Representative image for $n=21$ macropinosomes. Scale bar: $1 \mu \mathrm{m}$. c Localization dynamics of Phafin2 and Lifeact at macropinosomes. Lifeact localization shows partial temporal overlap with Phafin2 localization. $n=21$ macropinosomes, mean $+95 \% \mathrm{Cl}$. d Sequential images showing Phafin2 and Myo1E dynamics on a macropinosome. Representative image for $n=6$ macropinosomes. Scale bar: $1 \mu \mathrm{m}$. e Myo1E is recruited earlier than the initial Phafin2 bursts and shows partial temporal overlap during the first phase of Phafin2 recruitment. $n=6$ macropinosomes, mean $+95 \% \mathrm{Cl}$. $\mathbf{f}$ Sequential images of Phafin2 and FilaminA on a macropinosome. Representative images for $n=46$ macropinosomes. Scale bar: $1 \mu \mathrm{m}$. $\mathbf{g}$ Localization dynamics of Phafin 2 and FilaminA on macropinosomes. $n=46$ macropinosomes, mean $+95 \% \mathrm{Cl}$. h Sequential images showing that actin (visualized by Lifeact) forms a coat around Phafin2-positive macropinosomes. Gaps in the actin coat allow the passage of the Phafin2-labeled vesicle (arrow). Representative image for 18 cells. Scale bar: $1 \mu \mathrm{m}$. i Sequential images showing that FilaminA forms a coat around Phafin2-positive macropinosomes. Gaps in the FilaminA coat allow the passage of the Phafin2-labeled vesicle (arrow). Representative image for 20 cells. Scale bar: $1 \mu \mathrm{m}$. j SIM super-resolution image showing a Phafin2-positive macropinosome passing a gap in the actin cytoskeleton (arrow). Representative image for ten cells. Scale bar: $10 \mu \mathrm{m}$, inset $2 \mu \mathrm{m}$. $\mathbf{k}$ Schematic overview of the observed recruitment dynamics. Statistics source data for $\mathbf{b}, \mathbf{d}$, and $\mathbf{g}$ are provided in this paper.

plasma membrane (Fig. 4i-k). First, we tested if Phafin2 requires actin for its recruitment by treating cells expressing Phafin $2 \Delta \mathrm{C}$ with Latrunculin B (Supplementary Fig. S6a, b). This treatment did not affect Phafin2 $\Delta \mathrm{C}$ localization, indicating that Phafin2 localization is primarily driven by phosphoinositide binding and not by actin-binding.

We observed that overexpression of Phafin $2 \Delta \mathrm{C}$ triggered massive changes in cell morphology and the underlying actin cytoskeleton. Cells expressing Phafin $2 \Delta \mathrm{C}$ showed cell retractions and membrane blebs (Fig. 7e, Supplementary Movie S19). Membrane ruffling was largely suppressed; instead, flat membrane protrusions with multiple filopodia were formed (Fig. 7e). Cells expressing high levels of Phafin $2 \Delta \mathrm{C}$ were rounded and showed amoeboid-like cell migration (Supplementary Movie S20).

To test if this is caused by membrane-localized Phafin2, we treated cells expressing Phafin $2 \Delta \mathrm{C}$ with GSK-A1, which effectively removes Phafin $2 \Delta \mathrm{C}$ from the plasma membrane. The addition of GSK-A1 displaced Phafin $2 \Delta C T$ from the plasma membrane and suppressed the retraction and membrane blebbing
(Fig. 7f, g, Supplementary Movie S21), indicating that membranelocalized Phafin2, and not simple overexpression of Phafin $2 \Delta \mathrm{C}$, triggers these phenotypes.

In order to allow tight temporal control of these phenotypes, we used both rapamycin- and light-inducible dimerization ${ }^{35,36}$ to recruit wild-type Phafin 2 to the plasma membrane. Recruitment of Phafin 2 to the plasma membrane by either system triggered rapid cell retraction and membrane blebbing (Fig. 7h, i, j, Supplementary Fig. S6d-f, Supplementary Movies S22, S23, and S24). Live-cell imaging of cells expressing Lifeact-SNAP and light-recruitable Phafin2 showed that the membrane in these blebs was first devoid of actin (Fig. 7k,l), suggesting a delamination of the membrane from the actin cytoskeleton. Taken together, our data indicate that Phafin2 is recruited to membranes, where it can bind to actin and locally modulate actin dynamics.

Phafin2 allows macropinocytosis for nutrient scavenging in cancer cells. Finally, we addressed the importance of Phafin2 in a physiological process relying on macropinocytosis, the scavenging 
a

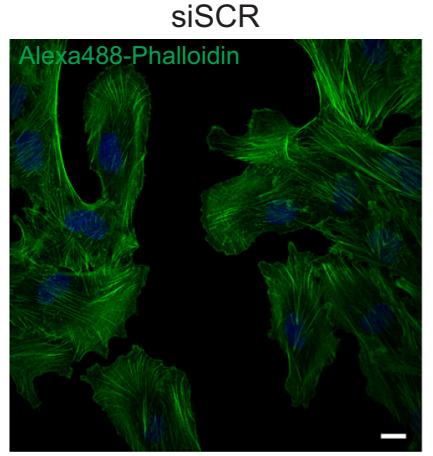

C
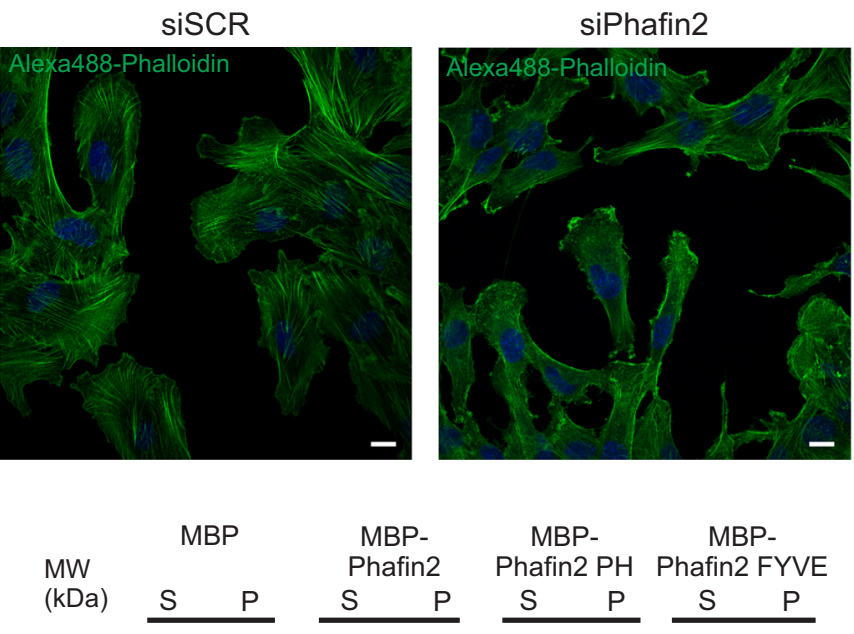

e
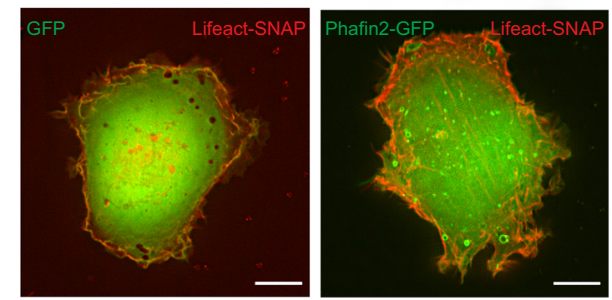

f

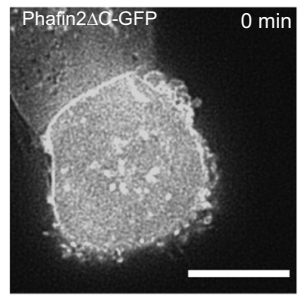

$\mathrm{h}$
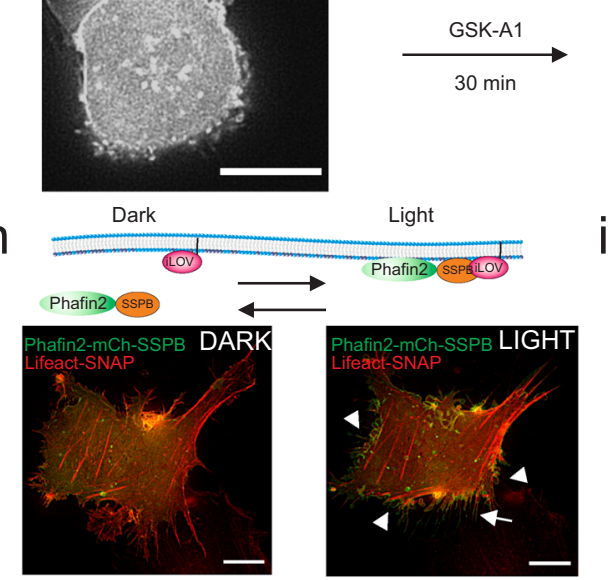

k

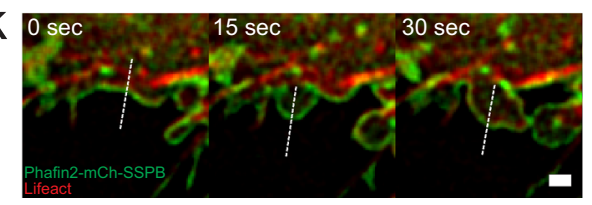

b

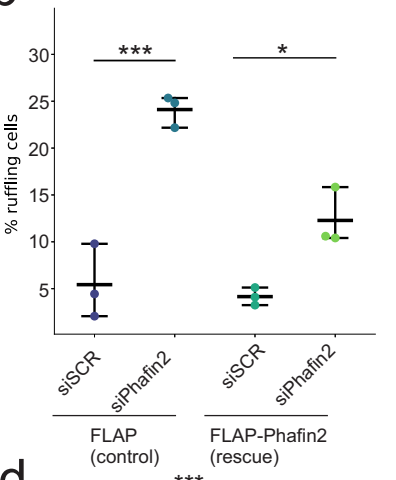

d
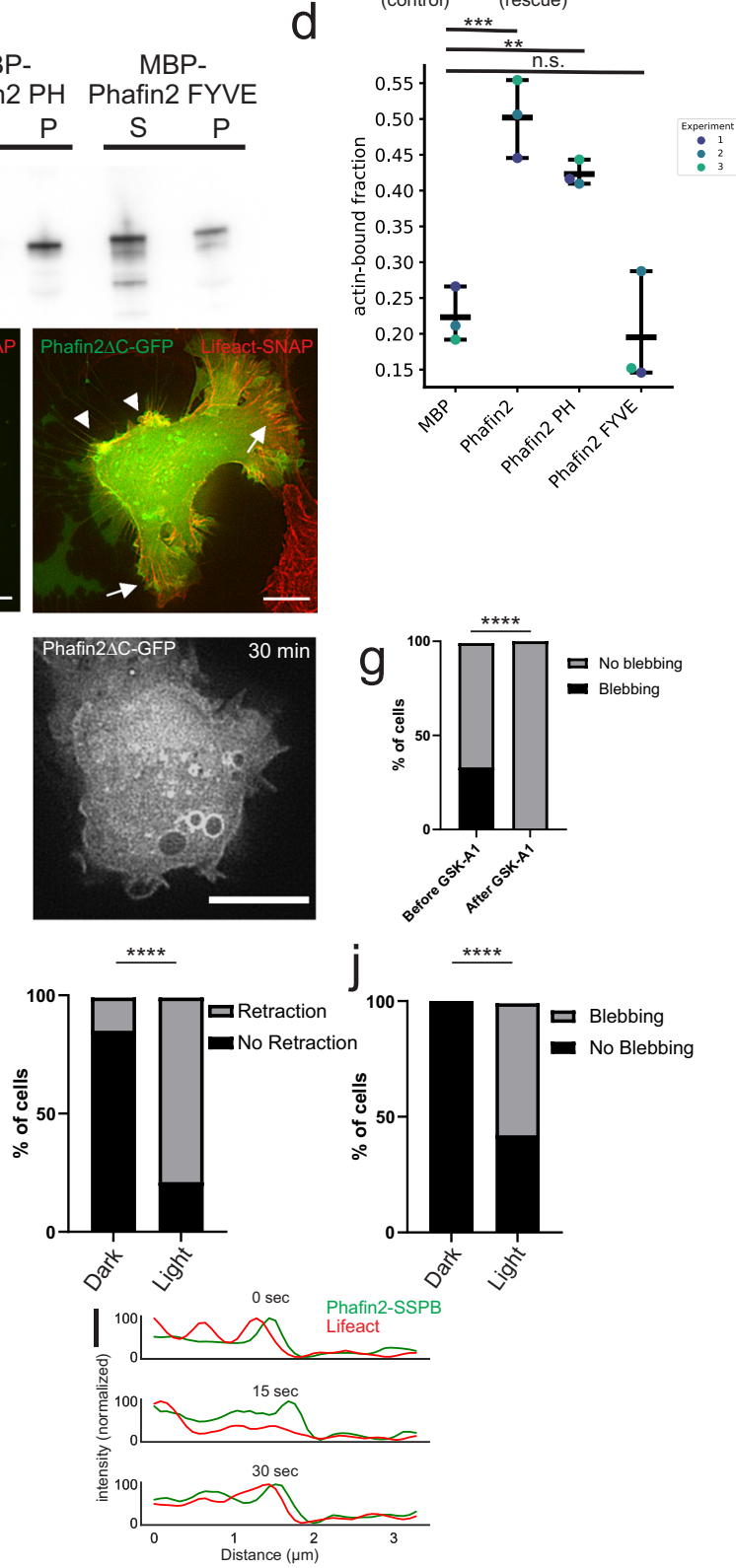

of extracellular proteins by KRAS-transformed cancer cells 4,37 . MIA-PACA2 cells-KRAS-transformed pancreatic cancer cellsexpress Phafin2 (Supplementary Fig. S7), and GFP-tagged Phafin2 labeled large vesicles and showed a biphasic localization pattern in these cells (Fig. 8a). We deleted Phafin2 in MIAPACA2 cells (Supplementary Fig. S7) and tested their ability to utilize extracellular proteins. Flow cytometry using self-quenched bovine serum albumin (DQ-BSA) showed strongly reduced BSA uptake in Phafin2 -/- cells (Fig. 8b). To test if Phafin2 is required to scavenge extracellular proteins under amino acid limiting conditions, we limited available glutamine and provided BSA as an alternative glutamine source ${ }^{4}$. While WT cells were able to utilize BSA, Phafin2 - - - cells were unable to scavenge extracellular BSA and showed reduced proliferation (Fig. 8c). 
Fig. 7 Phafin2 can bind to actin. a Confocal images showing the effect of Phafin2 depletion on the actin cytoskeleton. Representative image of the data scored in Fig. 7b. Scale bar: $10 \mu \mathrm{m}$. b Depletion of Phafin2 results in an increased number of cells with membrane ruffles, which can be rescued by expression of siRNA-resistant Phafin2. Data derived from high content imaging (2038, 1581, 1831, 2055 cells for siSCR, siPhafin2, siSCR(Rescue) and siPhafin2(Rescue) in total. $n=3$ experiments, mean $+95 \% \mathrm{Cl}$, two-sided $t$-test, $p=0.0017$ (Control), $p=0.017$ (Rescue). c Western blot showing in vitro actin-binding assays of MBP-Phafin2 and Phafin2 mutants. Phafin2 and the isolated Phafin2 PH domain co-sediment with actin. MBP or the isolated Phafin2 FYVE domain does not co-sediment. $\mathbf{d}$ Quantification of the in vitro actin-binding assays. $n=3$ experiments, mean $+95 \% \mathrm{Cl}$, ANOVA with Tukey's posttest, $p=0.0009$ (MBP vs Phafin2), $p=0.0073$ (MBP vs. Phafin2 PH), $p=0.91$ (MBP vs Phafin2 FYVE). e Images showing the effect of GFP, Phafin2-GFP, and Phafin2 $\Delta$ C-GFP expression in RPE1 cells expressing Lifeact-SNAP. Representative image of 30 cells each. Arrowheads indicate cell retractions/ membrane blebs; arrows indicate flat cell protrusions. Scale bar: $10 \mu \mathrm{m}$. $\mathbf{f}$ Time-lapse images of cells expressing Phafin $2 \Delta \mathrm{C}$ before and after incubation with the PI4K inhibitor GSK-A1. Scale bar: $10 \mu \mathrm{m}$. $\mathbf{g}$ Quantification of membrane blebbing after treatment of Phafin $2 \Delta \mathrm{C}$-expressing cells with the inhibitor GSKA1. $n=81$ cells. Two-sided Fisher's exact test, $p<0.0001$. h Time-lapse images of cells expressing light-dimerizable Phafin2 (Phafin2-mCherry-SSBP and Venus-iLID-CAAX) and Lifeact before and after light-induced plasma membrane recruitment. Arrowheads indicate membrane blebs, arrows cell retraction. Representative image of 25 cells. Scale bar: $10 \mu \mathrm{m}$. i Quantification of cell retraction after light-induced membrane recruitment of Phafin2. $n=28$ cells, twosided Fisher's exact test, $p<0.0001$. j Quantification of membrane blebbing after light-induced membrane recruitment of Phafin2. $n=28$ cells, two-sided Fisher's exact test, $p<0.0001$. k Time series showing a forming and retracting membrane bleb-visualized by membrane-recruited Phafin2-and Lifeact after Phafin2 membrane recruitment. Scale bar: $10 \mu \mathrm{m}$. I Line plots showing intensities of membrane-recruited Phafin2 and Lifeact over time. Statistics source data for $\mathbf{b}, \mathbf{d}, \mathbf{g}, \mathbf{i}, \mathbf{j}$, and $\mathbf{I}$ are provided in this paper.

a
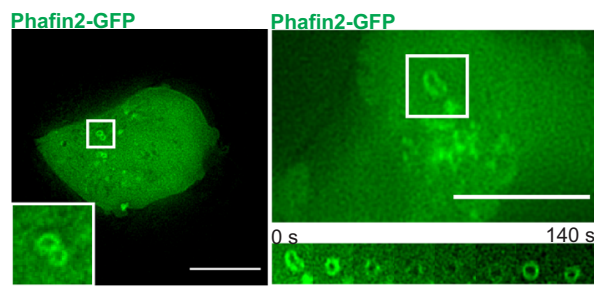

d

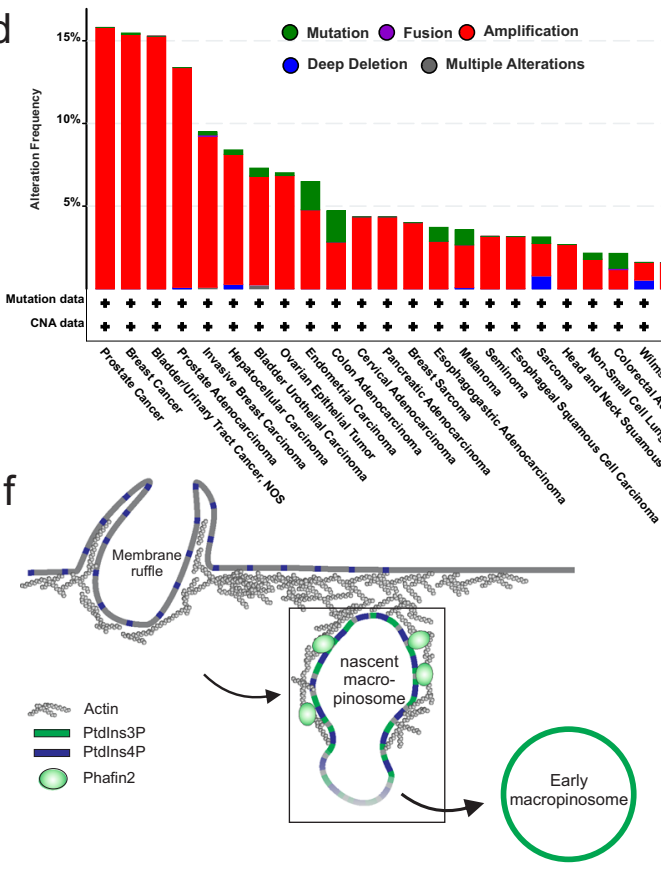

b

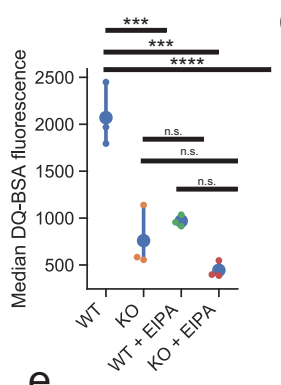

e

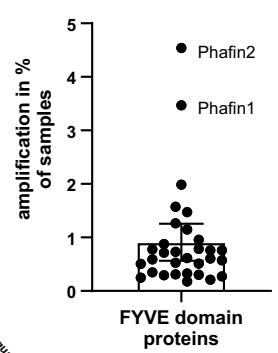

C
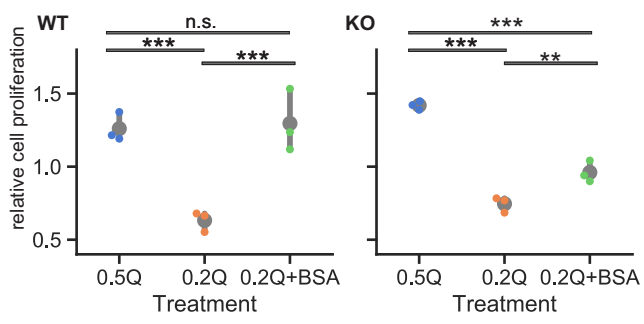

Fig. 8 Phafin2 is required for amino acid scavenging by cancer cells. Localization of Phafin2 in MIA-PACA2 cells. a Phafin2-GFP labels large vacuoles and shows biphasic localization to macropinosomes in MIA-PACA2 cells. Representative images from 10 cells. Scale bar: $10 \mu \mathrm{m}$. b Flow cytometry measurement of DQ-BSA by MIA-PACA2 cells. Deletion of Phafin2 strongly reduces BSA uptake. $n=3$ experiments, mean $+95 \% \mathrm{Cl}$, ANOVA with Tukey's post-test, $p=0.0002$. c MIA-PACA2 cells lacking Phafin2 are unable to scavenge extracellular nutrients. WT cells can scavenge extracellular proteins under amino acid limiting conditions. $\mathrm{KO}$ cells are unable to utilize extracellular proteins. $n=6$ experiments, shown are mean $+95 \% \mathrm{Cl}$, ANOVA with Tukey's post-test, $p=0.0001$. d Phafin2 is frequently amplified in cancer samples. A screen of the cBioportal cancer database shows frequent amplification of Phafin2 in cancer samples, whereas deletions or mutations are rare. e Phafin2 and the related protein Phafin1 are the only frequently amplified FYVE domain proteins. Shown is the frequency of amplification of 31 FYVE proteins in the curated dataset of non-redundant studies in the cBioPortal (representing 48834 non-redundant samples). Mean $+/-95 \% \mathrm{Cl}$. f Model of Phafin2 function on forming macropinosomes. Phafin2 binds to newly formed macropinosomes by coincidence sensing of Ptdlns3P and Ptdlns4P. By binding to filamentous actin, Phafin2 links the macropinosome membrane to the actin cytoskeleton. Turnover of phosphoinositides leads to a dissociation of Phafin2 from the macropinosome. Statistics source data for b, c, e are provided in this paper. 
These results indicate that Phafin2 is required for functional macropinocytosis in cancer cells. Screening the publicly available cBioportal cancer genome database ${ }^{38,39}$ for cancers with alterations in Phafin2 showed that Phafin2 is frequently amplified across multiple cancers, whereas deletions are very rare (Fig. $8 \mathrm{~d}$, e). This raises the possibility that moderate overexpression of Phafin2 is advantageous for cancer cells, potentially by supporting improved nutrient scavenging.

\section{Discussion}

In this study, we provide insights into the mechanisms of early macropinocytosis and its regulation by phosphoinositides. We identify Phafin2 as a phosphoinositide-binding regulator of macropinocytosis that marks a previously uncharacterized maturation stage of macropinosomes prior to their acquisition of early endosomal markers. Through binding to actin and causing its timely membrane dissociation, Phafin 2 promotes the transition of nascent macropinosomes through the dense subcortical actin network, thereby facilitating macropinocytosis. While Phafin2's function in macropinocytosis could be beneficial in antimicrobial defense, RAS-transformed cancer cells can co-opt Phafin2-dependent macropinocytosis for proliferation under lownutrient conditions.

After their formation, macropinosomes undergo drastic changes in their phosphoinositide composition. $\operatorname{Ptd} \operatorname{Ins}(3,4,5) \mathrm{P}_{3}$ is metabolized via $\operatorname{PtdIns}(3,4) \mathrm{P}_{2}$ and PtdIns3P to PtdIns $6,7,40,41$, which is then followed by generation of PtdIns3P directly after scission $^{41}$. In parallel, PtdIns4P is generated by dephosphorylation of PtdIns $(4,5) \mathrm{P}_{2}{ }^{8}$. So far, only few effectors of these transient lipid pools have been described. Phafin2 is a lipid coincidence sensor that recognizes transient PtdIns4P and PtdIns3P pools by its PH and FYVE domains. This is reminiscent of clathrin-mediated endocytosis, which also has been reported to require coincidence sensors for transient pools of PtdIns3P and PtdIns4 42,43 .

We find that membrane localization of Phafin2 is tightly controlled. The C-terminus of Phafin2 restricts its localization to forming macropinosomes and acts as a "noise suppressor", preventing spurious membrane association. Mechanistically, the Phafin2 C-terminus could-in line with a recent proposal ${ }^{27}-$ regulate the lipid-binding activity of the $\mathrm{PH}$ domain and, by this, prevent membrane binding. Alternatively, the negative charges could suppress Phafin2 binding to low concentration phosphoinositide pools, thus restricting Phafin2 localization to regions with high concentrations of phosphoinositides. Dephosphorylation of PtdIns $(4,5) \mathrm{P} 2$ to PtdIns4P by OCRL ${ }^{8}$ or other 5 phosphatases could provide a mechanism to simultaneously reduce negative charges on the membrane and produce PtdIns4P, which would participate in recruitment of Phafin2. Binding of Phafin2 could thus balance between available phosphoinositides and the charge of the plasma membrane, thereby allowing a finetuning of Phafin2 localization to membranes.

Phafin2 is critical for the early steps of macropinocytosis, likely by coordinating actin dynamics on newly formed macropinosomes by binding to actin. After scission from the membrane, newly formed vesicles are coated by a dense actin meshwork. Gaps in this network allow the macropinosome to escape its actin coat, thereby enabling maturation and progression into the endolysosomal pathway. While cells lacking Phafin2 still initiate macropinosomes, the formed vesicles remain coated in actin and frequently collapse and likely re-fuse with the plasma membrane. Phafin 2 could link the macropinosome membrane to the actin cytoskeleton, in line with findings that actin is required to overcome membrane tension during clathrin-mediated endocytosis ${ }^{44}$. Alternatively, the strong phenotype of membrane-localized
Phafin2 allows for the hypothesis that Phafin2 could directly modulate the actin cytoskeleton. Actin side-binding proteins can influence the rate of actin polymerization 45 and actin branching ${ }^{46}$, and high levels of Phafin 2 could locally regulate actin polymerization to allow uncoating of the macropinosome. The observed phenotypes are reminiscent of the phenotypes observed after decreasing Arp2/3 activity ${ }^{47,48}$, raising the possibility that Phafin 2 could locally inhibit actin branching. A similar mechanism has been reported for the protein CYRI-A, which transiently localizes to forming macropinosomes and locally suppresses actin dynamics by binding to $\operatorname{Racl}^{49}$.

Our collective findings suggest the following model (Fig. 8f): Scission from the plasma membrane results in a nascent macropinosome that is enmeshed in actin and trapped close to the plasma membrane. In order to mature, it has to escape this meshwork by removing actin nucleating factors from its limiting membrane and shed its actin coat. Transient production of PtdIns3P and PtdIns4P by dephosphorylation of PtdIns $(3,4) \mathrm{P}_{2}$ and PtdIns $(4,5) \mathrm{P}_{2}$ triggers recruitment of Phafin2, which couples the macropinosome membrane to the actin cytoskeleton. Asymmetric recruitment and release of Phafin2 by generation and dephosphorylation of PtdIns3P or PtdIns4P generate a Phafin2 gradient which directs forces on the vesicle.

Nutrient scavenging by macropinocytosis has emerged as an important amino acid supply route in KRAS*-transformed cancers 4,37 , and we find that loss of Phafin2 impairs this process. Phafin2 is frequently amplified in cancers, raising the possibility that higher Phafin2 levels provide a growth advantage for cancer cells, possibly by enhancing macropinocytosis. It will be interesting to learn if Phafin2 cooperates with KRAS* to enhance macropinocytosis or if amplification of Phafin2 could provide an alternative, independent way for cancer cells to upregulate nutrient scavenging by macropinosome formation. Phafin2 is highly expressed in immune cells-especially dendritic cells and CD19-positive B-cells ${ }^{50,51}$, suggesting that Phafin2-driven macropinocytosis could help these cells with antigen sampling.

\section{Methods}

Cell lines. Experiments were performed in hTert-RPE1 cells (ATCC CRL-4000), MIA-PACA2 cells (ATCC CRL-1420), HT1080 cells (ATCC CCL-121), and HeLa cells (CCL-2). Cell lines were obtained from ATCC. H2B-mCherry expressing cells were generated by transfection using pH2B-mCherrry-IRES-Neo, Neomycin selection and picking of single clones. Other stable cell lines were lentivirusgenerated pools. Third-generation lentivirus was generated using procedures and plasmids as previously described ${ }^{52}$. Briefly, tagged fusions of transgenes were generated as Gateway ENTRY plasmids using standard molecular biology techniques. From these vectors, lentiviral transfer vectors were generated by Gateway LR recombination into lentiviral destination vectors (Gateway-enabled vectors derived from pCDH-EF1a-MCS-IRES-PURO (SystemBiosciences)). VSV-G pseudotyped lentiviral particles were packaged using a third-generation packaging system (Addgene plasmids 12251, 12253, 12259). Cells were then transduced with low virus titers (multiplicity of infection $<1$ ) and stable expressing populations were generated by antibiotic selection. Detailed cloning procedures are available from the authors.

Stimulation of macropinocytosis in hTERT-RPE1 cells. We found that both size and initiation frequency of new macropinosomes in hTERT-RPE1 cells were strongly stimulated by addition of $50 \mathrm{ng} / \mathrm{ml}$ hepatocyte growth factor (HGF) (supplementary Fig. S5a, b, c). The effect of this stimulation persisted for up to $60 \mathrm{~min}$ after growth factor addition; we, therefore, performed all measurementsunless otherwise stated-in hTERT-RPE1 cells after addition of HGF. Imaging was limited to $40 \mathrm{~min}$ after stimulation to ensure consistent results.

Antibodies. The following antibodies were used: Phafin2 antibody, Sigma-Aldrich (HPA024829) (WB, 1:500). FilaminA antibody, Abcam (ab76289) (WB, 1:1000). Atto-488 conjugated anti-GFP nanobody, Chromotek (gba488) (IF, 1:100). $\gamma$ Tubulin antibody, Sigma-Aldrich (T5326)(WB, 1:10000). aTubulin antibody, Sigma-Aldrich (T5168) (WB, 1:20000). Rabankyrin-5 antibody (Abnova, H0005 1479-B01P) (IF, 1:100). MBP antiserum (New England Biolabs, E3080s) (WB,1:10000). Donkey anti-mouse Alexa555 (Molecular Probes. A31570)(1:500). 
Plasmids. pEYFP-Rabankyrin-5 was a gift from Marino Zerial; ${ }^{10}$ EYFP was exchanged with mCherry. The following plasmids were obtained from Addgene:

Myo1E-pmAppleC1 was a gift from Christien Merrifield (Addgene \#27698 ${ }^{30}$,), pH2B-mCherrry-IRES-Neo was a gift from Daniel Gerlich (Addgene \#21044 ${ }^{53}$ ), pDisplay-Phuji was a gift from Robert Campbell (Addgene \#61556 ${ }^{29}$,), pEGFPAKT-PH was a gift from Tobias Meyer (Addgene \#21218 ${ }^{54}$ ); EGFP was replaced with mCherry. pTagRFP-T-TAPP-2xPH was a gift from Tadaomi Takenawa ${ }^{55}$. pmCherry-FilaminA was a gift from Michael Davidson (Addgene plasmid \# 55047), pLifeact-mTurquoise2 was a gift from Dorus Gadella (Addgene plasmid \#36201); 56 mTurquoise 2 was replaced with mCherry and SNAP. pX458 and pX459 were a gift from Feng Zhang (Addgene plasmids \#48138 and $62988^{57}$ ). VenusiLID-CAAX and the SSPB-coding region were a gift from Brian Kuhlmann (Addgene $\# 60411$ and $\# 60415)^{35}$. Lyn-FRB-mCherry was a gift from Robin Irvine (Addgene \#38004) ${ }^{58}$. pET-His6-MBP was a gift of Scott Gradia (Addgene \# 29708).

All other plasmids were generated using standard cloning procedures. A list of plasmids used in this study can be found in Supplementary Table 1.

Transient transfection of cells. RPE1 cells were transfected with Fugene 6 using a ratio of 3:1 of reagent to DNA. In most cases, cells were transfected in MatTek $3.5 \mathrm{~cm}$ dishes using 6 ul of Fugene 6 and 2 ug of DNA. The medium was replaced $12 \mathrm{~h}$ after transfection to remove transfection reagent, and cells were imaged between $16 \mathrm{~h}$ and $20 \mathrm{~h}$ after transfection.

CRISPR/Cas9-mediated deletion of Phafin2. RPE1 cells deleted for Phafin2 were generated using CRISPR/Cas9. Guide RNAs were designed using Benchling software (www.benchling.com). For deletion of Phafin2, a guide RNA binding directly at the start codon in Exon 2 and one binding after Exon 2 was chosen (gRNA1: 5'AGGCTATTAGTGAAAGATGG-3'; gRNA2: 5'-TGGGAGTATTTAATCAGGTG$\left.3^{\prime}\right)$. As the whole ORF of Phafin2 is contained in this exon, we reasoned that the whole ORF should be excised. pX458-derived plasmids encoding both Cas9-2AGFP and the respective gRNA were transfected using Fugene $6.48 \mathrm{~h}$ post-transfection, GFP-positive cells were sorted and seeded out in several dilutions to obtain single colonies, which were picked and characterized. Clones lacking Phafin2 were identified by western blotting, and the introduced mutations were characterized by PCR, followed by cloning and sequencing. Primer sequences used for the characterization of knockout clones can be found in Supplementary Table S2. We established a Phafin2 clone lacking the whole Exon 2 in one allele and an inversion of the second allele, including a deletion of the start codon, resulting in a complete inactivation of Phafin2 (Supplementary Fig. S2). Deletion of Phafin2 in MIAPACA2 cells followed the same protocol, but a pX459-derived plasmid and puromycin selection instead of cell sorting were used.

siRNA-mediated depletion of Phafin2. siRNAs against Phafin2 were described before (Pedersen et al. $)^{11}$. The following sequences were used to deplete Phafin2: SiRNA 1 TGGTCAACCTTTAACTATA; siRNA2: GAAGCAAATACTAGAC GTA. For siRNA transfections, $50 \mathrm{nM}$ of siRNA was transfected using RNAiMax reagent. Transfected cells were analyzed $72 \mathrm{~h}$ after transfection; knockdown efficiency was verified by western blotting.

Live-cell imaging. All live-cell imaging-with the exception of the light sheet microscopy-was performed on a Deltavision OMX V4 microscope (GE Healthcare) equipped with $60 \times 1.42 \mathrm{NA}$ objective (Olympus), three water cooled PCO.edge sCMOS cameras, a solid-state light source, and a laser-based autofocus. Environmental control was provided by a heated stage and an objective heater (2020 Technologies). Cells were imaged in Live-Cell Imaging buffer (Invitrogen) supplemented with $20 \mathrm{mM}$ glucose. To enhance the rate of macropinosome formation, cells were stimulated by adding HGF $(50 \mathrm{ng} / \mathrm{ml})$ directly before imaging. For imaging of Lifeact-SNAP, cells were stained with SiR647-SNAP (New England Biolabs) according to the manufacturer's protocol. Images were deconvolved using softWoRx software and processed in Fiji ${ }^{59,60}$.

TIRF microscopy. TIRF microscopy was performed with the Ring-TIRF mode of the OMX V4 system described above, using a $60 \times 1.49$ NA objective (Olympus), using $488 \mathrm{~nm}$ and $561 \mathrm{~nm}$ laser lines. All other imaging conditions were as described above.

High-resolution light-sheet microscopy. Light-sheet microscopy was performed using a custom-built oblique plane microscope. The microscope layout followed the general plan for a stage scanning OPM microscope described in Sapoznik et al. ${ }^{61}$. The microscope was built around the ASI modular microscope platform. The inverted microscope consisted of an ASI MIM microscope body, an ASI FTP Z stage and a stage scanning optimized ASI X/Y stage (ASI imaging). Environmental control was provided by an Okolabs stage incubator. A $60 \times 1.3$ NA silicon oil immersion lens (Olympus) served as the primary objective. ASI cage elements were used to construct the rest of the optical train, consisting of a $300 \mathrm{~mm}$ tube lens (TL1), a $357 \mathrm{~mm}$ tube lens (Tl2), and a $40 \times 0.95 \mathrm{NA}$ air remote objective (Nikon). The image generated by the remote objective was collected by a bespoke tertiary objective (AMS-AGY v1.0, Special Optics) and focused by a $250 \mathrm{~mm}$ tube lens
(TL3) on the sensor of an Andor Zyla 4.2 camera, resulting in a final pixel size of $91 \mathrm{~nm}$. The excitation beam was coupled into the light path by a dichroic mirror placed between TL2 and the remote objective. We used a gaussian light sheet generated by an ASI light sheet generator (ASI Imaging) equipped with a cylindrical lens, coupled to a Toptica laser source (Toptica) with 405, 488, 561, and $647 \mathrm{~nm}$ laser lines. Image acquisition was performed by stage scanning. All hardware was synchronized by an ASI Tiger controller (ASI Imaging) and controlled by the ASI diSPIM plugin in MicroManager ${ }^{62}$. Deconvolution and deskewing were performed using the LLSPy software package (https://github.com/ tlambert03/LLSpy/), the resulting images were visualized using Imaris.

Structured illumination microscopy. Three dimensional SIM imaging was performed on Deltavision OMX V4 microscope with an Olympus x60 NA 1.42 objective and three PCO.edge sCMOS cameras and $488 \mathrm{~nm}$ and $568 \mathrm{~nm}$ laser lines. Cells were illuminated with a grid pattern, and for each image plane, 15 raw images (five phases and three rotations) were acquired. Super-resolution images were reconstructed from the raw image files, aligned and projected using Softworx software (Applied Precision, GE Healthcare). Images were processed in ImageJ/Fiji 60 .

Release of mitochondrially tethered MTM1 using the RCD1 reversible dimerization system. RPE1 cells stably expressing Phafin2-GFP were transfected with a Tom20 mitochondrial targeting signal (MTS)-mTagBFP2-3xSNAPtag construct and an mCherry-FKBP-MTM1 (or MTM1 C375S) construct and incubated overnight in media containing $1 \mu \mathrm{M}$ rCD1 (Sirius Fine Chemicals). rCD1 caused heterodimerization of the FKBP and SNAPtag moieties, anchoring MTM1 to the mitochondria. Live-cell imaging was performed to visualize Phafin2 recruitment to macropinosomes. During live-cell imaging, 1uM FK506 was added to release MTM1 into the cytoplasm, and this was monitored by mCherry fluorescence.

Rapamycin-controlled membrane recruitment of Phafin2. Rapamycin-mediated FKBP-FRB dimerization was used for acute membrane recruitment of Phafin $2^{36}$ Cells were transfected with Phafin2-GFP-FKBP and Lyn-FRB-mCherry. Dimerization was triggered by the addition of $250 \mathrm{nM}$ of rapamycin.

Optogenetic membrane recruitment of Phafin2. The iLID light-inducible dimerization system was used for acute, light-controlled membrane recruitment of Phafin $2^{35}$. Cells were transfected with Phafin2-mCherry-SspB and Venus-iLIDCAAX. Dimerization was triggered by excitation of the sample with the FITC channel of the OMX widefield light source.

Bacterial infection assays. Infection experiments were performed using Salmonella enterica serovar Typhimurium SL1344 expressing the pGG2 (dsRed) plasmids. The day before the infection, bacteria were grown overnight in $3 \mathrm{~mL}$ Lysogeny broth medium supplemented with $0.3 \mathrm{M} \mathrm{NaCl}$ at $37^{\circ} \mathrm{C}$ in an orbital shaker. Growth media was supplemented with $50 \mathrm{mg} / \mathrm{mL}$ ampicillin. On the day of the infection, bacteria were subcultured at a 1:21 dilution in Lysogeny broth supplemented with $0.3 \mathrm{M} \mathrm{NaCl}$ and incubated at $37^{\circ} \mathrm{C}$ for $3 \mathrm{~h}$ (i.e., until late exponential phase). Bacteria were then washed once and resuspended in EM buffer $\left(120 \mathrm{mM} \mathrm{NaCl}, 7 \mathrm{mM} \mathrm{KCl}, 1.8 \mathrm{mM} \mathrm{CaCl}_{2}, 0.8 \mathrm{mM} \mathrm{MgCl}_{2}, 5 \mathrm{mM}\right.$ glucose, $25 \mathrm{mM}$ HEPES, $\mathrm{pH}$ 7.3). Bacterial concentration was determined using OD600, and bacteria were diluted to the desired multiplicity of infection in warm EM buffer. For time-lapse experiments, fields of view were selected at the microscope in a $37^{\circ} \mathrm{C}$ chamber. Then, the bacteria were added to the cells before the beginning of the acquisition. Time-lapse experiments were performed on an inverted Deltavision epifluorescence microscope (GE Healthcare) and a 60x oil objective. Images were deconvolved using softWoRx software

Immunostaining and stimulation of macropinocytosis. siRNA treated cells grown on glass coverslips were stimulated with HGF $(50 \mathrm{ng} / \mathrm{ml})$ for 15 minutes in full medium (DMEM-F12, 10\% FCS, Pen/Strep), fixed with 3\% formaldehyde (Polysciences, 18814) for $15 \mathrm{~min}$ on ice, and permeabilized with $0.05 \%$ saponin (Sigma-Aldrich, S7900) in PBS. Fixed cells were then stained with a mouse antiRabankyrin-5 antibody (Abnova, cat no: H0005 1479-B01P) at room temperature for $1 \mathrm{~h}$, washed in PBS/saponin, stained with a fluorescently labeled secondary antibody (donkey anti-mouse Alexa555, Molecular Probes CatNo.: A31570) for $1 \mathrm{~h}$, washed in PBS and mounted with Mowiol (Sigma-Aldrich) containing $2 \mu \mathrm{g} / \mathrm{ml}$ Hoechst 33342 (Thermo Fisher Scientific, H3570). Alexa488-Phalloidin (cat no: A12379) or Alexa647-Phalloidin (cat no: A22287) were used to detect filamentous actin.

High content imaging and analysis of Rabankyrin- 5 dots and actin ruffling cells. Widefield images of siRNA transfected cells labeled for endogenous Rabankyrin-5 or filamentous actin (Alexa488-Phalloidin) were acquired automatically by an Olympus ScanR high content microscopy system using a UPLSAPO 40x objective and analyzed automatically by the ScanR software. After background subtraction, dots of Rabankyrin-5 $>30$ pixels were segmented 
automatically by the ScanR software based on an intensity threshold, and the number of dots per cell was measured. The total number of cells was quantified automatically by the detection of Hoechst nuclear stain. Identical settings were used for all conditions within an experiment. Cells with prominent actin ruffling were scored manually.

Confocal fluorescence microscopy. Confocal images were obtained using LSM710 confocal microscope (Carl Zeiss) equipped with an Ar-laser multiline $(458 / 488 / 514 \mathrm{~nm})$, a DPSS-561 $10(561 \mathrm{~nm})$, a laser diode 405-30 CW $(405 \mathrm{~nm})$, and a HeNe laser $(633 \mathrm{~nm})$. The objective used was a Plan-Apochromat $63 \times / 1.40$ oil DIC III (Carl Zeiss). Image processing was performed with basic software ZEN 2010 (Carl Zeiss) and Fiji software.

Measurement of dextran and DQ-BSA uptake by flow cytometry. Fluid-phase uptake was assayed by measuring fluorescent dextran uptake using flow cytometry. Fluorescent dextran Alexa Fluor $48810 \mathrm{kDa}$ was added to cells $(0,5 \mathrm{mg} / \mathrm{ml})$ stimulated with $\mathrm{HGF}$ and cells were incubated for $20 \mathrm{~min}$ at $37^{\circ} \mathrm{C}$. Cells were then washed five times with prewarmed medium, trypsinized and dextran fluorescence was measured by flow cytometry. Alternatively, uptake was measured with selfquenched DQ-BSA. Cells were incubated with $10 \mu \mathrm{g} / \mathrm{ml}$ DQ-BSA for $30 \mathrm{~min}$, washed, and chased for $1 \mathrm{~h}$. Cells were then trypsinized, and DQ-BSA fluorescence was measured by flow cytometry. All flow cytometry measurements were performed using an LSR II flow cytometer equipped with 488 and $561 \mathrm{~nm}$ laser lines. For all flow cytometry measurements, live cells and single cells were gated, and the dextran/DQ-BSA fluorescence was measured. An exemplary gating scheme is shown in Supplementary Fig. S8.

\section{Dextran fluorescence by microscopy. Cells of the indicated genotypes were} seeded in glass-bottomed MatTek dishes. The media was replaced by prewarmed media containing $0.5 \mathrm{mg} / \mathrm{ml}$ dextran-Alexa Fluor $488(10 \mathrm{kDa})$ and $50 \mathrm{ng} / \mathrm{ml} \mathrm{HGF}$. Cells were incubated at $37^{\circ} \mathrm{C}$ for 30 mins. After the incubation, cells were quickly washed four times with prewarmed media, once with phosphate-buffered saline, and fixed for $10 \mathrm{~min}$ at room temperature using $4 \%$ paraformaldehyde in PBS. The cells were gently washed three times in PBS and the plasma membrane labeled with Wheat Germ Agglutinin-Alexa Fluor 647 (Molecular Probes) at $5 \mu \mathrm{g} / \mathrm{ml}$ for 10 mins in PBS. The cells were washed twice, the nuclei labeled with Hoechst 33342 (Molecular Probes), and imaged in PBS. Image z-stacks of $6 \mu \mathrm{m}$ were acquired at an interval of $250 \mathrm{~nm}$ and deconvolved. One cell was measured per field of view acquired (the field of view was typically only large enough to fully fit one cell). Image stacks were z-projected using the sum of intensities. Cell outlines were segmented in an ImageJ script using the plasma membrane marker as a guide. Background values (compensation for residual nonspecific dextran and imperfect deconvolution) were obtained from an ROI in each image, excluding all cells segmented. Where the ImageJ script failed to generate a good quality segmentation, we manually generated the ROIs and took background values from a $100 \times 100$ pixel square outside cells. The intracellular dextran fluorescence was computed by subtracting the background from mean fluorescence within the cell and multiplying by cell area to obtain the integrated intensity of the cell. Measurement scripts are available at https://github.com/koschink/Phafin2.

Measurement of cell proliferation under amino acid limiting conditions. MIAPACA2 cells, either WT or Phafin2 - / - cells, were seeded in 6-well plates $(50,000$ cells/well). For each experiment, two technical replicates were used to minimize measurement errors. One day after seeding, the medium was changed to LGlutamine-free DMEM supplemented with $10 \%$ dialyzed FBS and $0.5 \mathrm{mM}$ Glutamine (control), $0.2 \mathrm{mM}$ Glutamine (starvation), or $0.2 \mathrm{mM}$ Glutamin and $2 \%$ BSA (Starvation supplemented with protein). The medium was changed every second day, and after 5 days, cells were trypsinized, resuspended in $1 \mathrm{ml}$ of medium, and counted using a Coulter counter.

Membrane scission assays. Membrane scission was assayed by measuring the fluorescence of the $\mathrm{pH}$-sensitive RFP $\mathrm{pHuji}$ in response to $\mathrm{pH}$ changes. $\mathrm{pHuji}$ localized in sealed vesicles does not respond to changes of the extracellular $\mathrm{pH}$, whereas surface-exposed fluorophores are readily quenched. Cells stably expressing Phafin2-GFP were seeded in MatTek dishes and transfected with pDisplay-pHuji. Imaging was performed in Live-Cell Imaging buffer (Invitrogen) supplemented with $20 \mathrm{mM}$ glucose. After being placed on the microscope stage, a perfusion pencil (Autom8 Scientific) was placed directly on top of the cell using a micromanipulator. Short bursts of imaging buffer $(140 \mathrm{mM} \mathrm{NaCl}, 2.5 \mathrm{mM} \mathrm{KCl}, 1.8 \mathrm{mM}$ $\mathrm{CaCl} 2,1.0 \mathrm{mM} \mathrm{MgCl} 2,20 \mathrm{mM}$ PIPES or HEPES) buffered to $\mathrm{pH} 5.5$ and 7.5 with PIPES and HEPES, respectively, were alternatingly perfused in $5 \mathrm{~s}$ intervals using a gravity driven perfusion apparatus (Warner). All solutions were heated using an inline heating element (Warner). Perfusion intervals were controlled by an Arduino microcontroller using PyFirmata and custom Python scripts (available at https:// github.com/koschink/Arduino_Perfusion). Alternatively, instead of using $\mathrm{pH}$ changes, cells expressing the calcium sensor GCAMP6 were perfused with imaging buffers containing EGTA or Calcium using the same setup. The time point of sealing was manually determined by monitoring individual vesicles, and Phafin2 intensity was tracked as described below.
Protein-lipid overlay assays. In vitro lipid-binding activities of Phafin2 $\mathrm{PH}$ and FYVE were determined by protein-lipid overlay assays ${ }^{17}$. Protein expression, purification, and lipid-overlay assays were performed as previously described ${ }^{63}$.

Protein expression and purification. Recombinant GST- and His-MBP proteins were produced in E.coli Rosetta2(DE3) cells. Cells were grown in ZYM505 medium, and protein expression was induced by the addition of $250 \mu \mathrm{M}$ IPTG at $20^{\circ} \mathrm{C}$ overnight.

Protein purification of GST fusion proteins was performed as described ${ }^{63}$. Purification of His-MBP fusion proteins was performed as follows. Cells were harvested and resuspended in lysis buffer $(50 \mathrm{mM}$ Tris, $150 \mathrm{mM} \mathrm{NaCl}, 10 \mu \mathrm{M}$ $\mathrm{ZnCl}_{2}, 1 \mathrm{mM}$ TCEP, $20 \mathrm{mM}$ imidazole) supplemented with Complete protease inhibitors (Roche) and lysed using homogenization in an LM20 microfluidizer. Lysates were cleared by centrifugation. The cleared lysates were then loaded on HisPur columns (Pierce), and unbound proteins were removed by washing with lysis buffer. The bound protein was eluted with elution buffer $(50 \mathrm{mM}$ Tris, $300 \mathrm{mM} \mathrm{NaCl}, 10 \mu \mathrm{M}$ ZnCl2, $1 \mathrm{mM}$ TCEP, $250 \mathrm{mM}$ imidazole). Protein containing fractions were pooled, dialyzed against either liposome buffer $(50 \mathrm{~m}$ HEPES, $150 \mathrm{mM} \mathrm{KCL}, 100 \mu \mathrm{M} \mathrm{ZnCl} 2,1 \mathrm{mM}$ TCEP) or actin assay buffer $(50 \mathrm{mM}$ Tris, $100 \mathrm{mM} \mathrm{NaCl}, 100 \mu \mathrm{M} \mathrm{ZnCl} 2,1 \mathrm{mM}$ TCEP) overnight, snap frozen, and stored at $-80^{\circ} \mathrm{C}$.

Liposome flotation assays. Liposome flotation assays were performed similar to published procedures 21,64 . We generated liposomes with an endosome-like lipid composition and doped them with different concentrations of phosphoinositides. We chose $0.5 \%$ PtdIns3P and 5\% PtdIns4P to simulate the high levels of plasma membrane-derived PtdIns4P and low levels of a phosphatase-generated PtdIns3P. PI was used as balance in liposomes doped with a single species and as negative control. Lipids (47\% PC, 25\% PE, 9\% Cholesterol, 10\% PS, 5\% PI (Avanti Polar Lipids) and $0.2 \%$ NBD-PE (Thermo Fisher), all \% are molar \%) were dissolved in Chloroform. Then, $0.5 \%$ PtdIns3P and 5\% PtdIns4P were added. In case that only one phosphoinositide was used, PI was used as balance. Liposomes containing PI only were used as negative control. The solvent was evaporated under a nitrogen stream and the lipid film dried under vacuum. The dried lipids were rehydrated in liposome buffer (50 m HEPES, $150 \mathrm{mM} \mathrm{KCL}, 100 \mu \mathrm{M} \mathrm{ZnCl} 2,1 \mathrm{mM}$ TCEP) and multilamellar liposomes were formed by five freeze-thaw cycles. The resulting liposome mixture was then extruded by 11 passages through a $400 \mathrm{~nm}$ filter membrane. Recombinant MBP-Phafin2 and Phafin2 mutants were added to liposomes ( $1 \mathrm{mM}$ lipid) in a final volume of $150 \mu \mathrm{l}$ and incubated for $20 \mathrm{~min}$ at RT. To this mixture, $100 \mu \mathrm{l}$ of $75 \%$ Sucrose in liposome buffer was added, resulting in a $30 \%$ sucrose solution. This fraction was overlaid with $200 \mu \mathrm{l} 25 \%$ sucrose in liposome buffer and $100 \mu \mathrm{l}$ of liposome buffer without sucrose. The sample was then centrifuged at 55,000 r.p.m. (240,000 g) in a Beckman swing rotor (TLS 55) for $1 \mathrm{~h}$. Successful liposome flotation was verified by visualizing NBD-PE fluorescence using a "Safelight" gel imager, and fractions ( $250 \mu \mathrm{l}$ (bottom), $200 \mu \mathrm{l}$ (middle), and $100 \mu \mathrm{l}$ (top)) were collected from the bottom. $25 \mu \mathrm{l}$ of the top fraction was separated using SDS-PAGE and visualized by Coomassie Brilliant Blue staining. Gels were recorded using an "Odyssey" gel imaging device (LI-COR Biotechnology). Intensities were measured using Fiji and plotted using the Seaborn Python package.

Actin spin-down assays. The binding of Phafin 2 and Phafin 2 mutants to filamentous actin was measured using actin spin-down assays. His-MBP-Phafin2, Phafin2 subdomains (His-MBP-PH, His-MBP-FYVE), and isolated His-MBP were expressed as fusions in E.coli as described above. Directly before use, proteins were centrifuged for $1 \mathrm{~h}$ at $150,000 \mathrm{~g}$ to remove insoluble or aggregating proteins. Spindown assays were performed with the Cytoskeleton actin spin-down kit according to the manufacturer's instructions. Polymerized non-muscle actin was mixed with His-MBP-fusion proteins in actin polymerization buffer (final volume $100 \mu \mathrm{l}$ ). The dialysis buffer was used as balance. After incubation at RT for $1 \mathrm{~h}$, filamentous actin was sedimented by ultracentrifugation $(1.5 \mathrm{~h}, 150,000 \mathrm{~g})$, and both pellet and supernatant fraction were analyzed by SDS-PAGE. MBP fusion was detected by western blotting with an anti-MBP antibody. Intensities were measured using Fiji and plotted using the Seaborn Python package. We quantified the intensity of the MBP fusion proteins in both the supernatant and pellet fractions $(=100 \%)$ and plotted the fraction bound to actin in the pellet.

Image pre-processing and data analysis. All live-cell images were deconvolved using Softworx (GE Healthcare) prior to analysis and presentation. All further image analysis and measurement steps were performed in FIJI-either by manual scoring or using custom Jython scripts. Measurement scripts are available at https://github.com/koschink/Phafin2.

Quantification of macropinosome fusion. Macropinosome fusion was quantified by manually tracking individual macropinosomes from their formation to the endosomal phase and visually scoring fusion events.

Tracking of macropinosomes. Newly formed macropinosomes were identified in time-lapse movies and manually tracked by using Phafin 2 or membrane markers as 
reference. For each time point, a region of their limiting membrane was marked as the region of interest (ROI). Fluorescence intensity of a circular ROI (10 pixel diameter) surrounding the marked region was quantified in all image channels by a Jython script and measurements were exported for further analysis. All further processing steps were performed using Python scripts and the "Pandas" data analysis package. Individual tracks of macropinosomes were temporally aligned by identifying the highest Phafin2 intensity in the first 15 frames of each track (corresponding to the early phase of Phafin2 localization) and aligning all tracks to this maximum. The measurements of each track were normalized, and the mean value and the $95 \%$ confidence interval were plotted for presentation using the Seaborn Python package. Both measuring and post-processing analysis scripts are available at https://github.com/koschink/Phafin2.

Quantification of macropinosome formation. To quantify newly formed macropinosomes (Fig. 5b, c), Phafin2 KO cells were mixed with WT cells stably expressing H2B-mCherry and transfected with GFP-2xFYVE and Lifeact-SNAP. Cells were then stimulated with HGF, and ruffling cells were imaged for 20 mins ( $30 \mathrm{~s} /$ frame). Choosing of the cells was blinded in regard to the genotype (WT cells were labeled by H2B-mCherry). Macropinosome formation was then manually quantified by scoring the number of newly formed macropinosomes-large $(>1 \mu \mathrm{m}$ diameter) 2xFYVE positive vesicles-within the imaging period.

Quantification of successful vs. aborted macropinosome formation. Stable cell lines (WT and Phafin2 KO) expressing mNG-2xFYVE and MyrPalm-mCherry were stimulated with HGF and imaged ( $5 \mathrm{~s} /$ frame). Newly forming macropinosomes were identified as cup-shaped membrane structures using the MyrPalmmCherry channel. Cup-shaped membrane structures successfully gaining mNG$2 \mathrm{xFYVE}$ and $>1 \mu \mathrm{m}$ in diameter were scored as successfully formed macropinosomes.

Automated measurements of macropinosome size. In order to measure the size of newly formed macropinosomes, individual 2xFYVE labeled vesicles were tracked and their size was measured using Trackmate ${ }^{65}$ and a custom FIJI script. For each individual track, we extracted the size of the vesicle at the first time point using a custom Python script. We reasoned that extracting the first time point would provide us with the size of macropinosomes prior to fusion and fission events. We filtered out vesicles $<0.64 \mu \mathrm{m}^{2}$ to select for macropinosomes. We then plotted the mean size of newly formed macropinosomes per cell and the size of individual macropinosomes using Seaborn. Custom measurement and analysis scripts are available at https://github.com/koschink/Phafin2.

Statistical analysis. Statistical analysis was performed using Graphpad Prism. Student's $t$-test was used as a measure for statistical significance when comparing two groups, ANOVA was used when comparing multiple groups. Data are always presented as mean $\pm 95 \% \mathrm{CI}, p$-values $<0.05$ were considered to be statistically significant. Sample sizes and $p$-values are reported in the figure legends. For visual annotation of statistical significance in graphs, the following nomenclature was used: $* p<0.05,{ }^{*} p<0.01,{ }^{* * *} p<0.001$, n.s. not statistically significant. Detailed statistical information for ANOVA is provided as ANOVA tables in the source data. In the case of some flow cytometry-based Dextran uptake assays (Supplementary Fig. S3e), measurements were normalized by setting the control measurements to one and a one-sample t-test was used to account for the lack of variability of the control. Categorical data were analyzed by Fisher's exact test. The correlation between Filamin A removal and macropinosome survival was tested using Spearman's correlation (Supplementary Fig. S5c).

Software availability statement. Custom software scripts are available at https:// github.com/koschink/Phafin2 (https://doi.org/10.5281/zenodo.5512979) ${ }^{66}$, control software for the perfusion setup is available at https://github.com/koschink/ Arduino_Perfusion.

Reporting Summary. Further information on research design is available in the Nature Research Reporting Summary linked to this article.

\section{Data availability}

All data shown and used to generate plots, as well as detailed statistical information, accompanies this manuscript in the source data file. Uncropped western blots and gels are shown in Supplementary Fig. S9. Underlying image data are available from the corresponding authors (H.St, K.O.S) upon request. Expression data for Phafin2 is available at the public BioGPS database (https://biogps.org/gene/79666/), the cancer copy number alteration data shown in Fig. $8 \mathrm{~d}$ and $8 \mathrm{e}$ are available at the cBioPortal database (https://www.cbioportal.org/). Source data are provided with this paper.

Received: 5 July 2021; Accepted: 8 October 2021; Published online: 12 November 2021

\section{References}

1. Swanson, J. A. Shaping cups into phagosomes and macropinosomes. Nat. Rev. Mol. Cell Biol. 9, 639-649 (2008).

2. King, J. S. \& Kay, R. R. The origins and evolution of macropinocytosis. Philos. Trans. R. Soc. Lond. B Biol. Sci. 374, 20180158 (2019).

3. Mercer, J. \& Helenius, A. Virus entry by macropinocytosis. Nat. Cell Biol. 11, 510-520 (2009).

4. Commisso, C. et al. Macropinocytosis of protein is an amino acid supply route in Ras-transformed cells. Nature 497, 633-637 (2013).

5. Bohdanowicz, M. \& Grinstein, S. Role of phospholipids in endocytosis, phagocytosis, and macropinocytosis. Physiological Rev. 93, 69-106 (2013).

6. Veltman, D. M. et al. A plasma membrane template for macropinocytic cups. eLife 5, e20085 (2016).

7. Maekawa, M. et al. Sequential breakdown of 3-phosphorylated phosphoinositides is essential for the completion of macropinocytosis. Proc. Natl Acad. Sci. USA 111, E978-E987 (2014).

8. Maxson, M. E., Sarantis, H., Volchuk, A., Brumell, J. H. \& Grinstein, S. Rab5 regulates macropinocytosis by recruiting the inositol 5-phosphatases OCRL and Inpp5b that hydrolyse PtdIns(4,5)P2. J. Cell Sci. 134, jcs252411(2021).

9. Zoncu, R. et al. A phosphoinositide switch controls the maturation and signaling properties of APPL endosomes. Cell 136, 1110-1121 (2009).

10. Schnatwinkel, C. et al. The Rab5 effector Rabankyrin-5 regulates and coordinates different endocytic mechanisms. PLoS Biol. 2, E261 (2004).

11. Pedersen, N. M. et al. The PtdIns3P-binding protein Phafin 2 mediates epidermal growth factor receptor degradation by promoting endosome fusion. Traffic 13, 1547-1563 (2012).

12. Zacharias, D. A., Violin, J. D., Newton, A. C. \& Tsien, R. Y. Partitioning of lipid-modified monomeric GFPs into membrane microdomains of live cells. Science 296, 913-916 (2002).

13. Fredlund, J. et al. The entry of Salmonella in a distinct tight compartment revealed at high temporal and ultrastructural resolution. Cell. Microbiol. https://doi.org/10.1111/cmi.12816 (2018).

14. Stevenin, V. et al. Dynamic growth and shrinkage of the salmonellacontaining vacuole determines the intracellular pathogen niche. Cell Rep. 29, 3958-3973 e3957 (2019).

15. Miaczynska, M. et al. APPL proteins link Rab5 to nuclear signal transduction via an endosomal compartment. Cell 116, 445-456 (2004).

16. Yeo, J. C., Wall, A. A., Luo, L. \& Stow, J. L. Rab31 and APPL2 enhance FcgammaR-mediated phagocytosis through PI3K/Akt signaling in macrophages. Mol. Biol. cell 26, 952-965 (2015).

17. Dowler, S., Kular, G. \& Alessi, D. R. Protein lipid overlay assay. Science's STKE: signal Transduct. Knowl. Environ. 2002, pl6 (2002).

18. Matsuda-Lennikov, M. et al. Lysosomal interaction of Akt with Phafin2: a critical step in the induction of autophagy. PloS One 9, e79795 (2014).

19. Raiborg, C., Bache, K. G., Mehlum, A., Stang, E. \& Stenmark, H. Hrs recruits clathrin to early endosomes. EMBO J. 20, 5008-5021 (2001).

20. Gillooly, D. J. et al. Localization of phosphatidylinositol 3-phosphate in yeast and mammalian cells. EMBO J. 19, 4577-4588 (2000).

21. Sneeggen, M. et al. WDFY2 restrains matrix metalloproteinase secretion and cell invasion by controlling VAMP3-dependent recycling. Nat. Commun. 10, 2850 (2019).

22. Ronan, B. et al. A highly potent and selective Vps34 inhibitor alters vesicle trafficking and autophagy. Nat. Chem. Biol. 10, 1013-1019 (2014).

23. Taylor, G. S., Maehama, T. \& Dixon, J. E. Myotubularin, a protein tyrosine phosphatase mutated in myotubular myopathy, dephosphorylates the lipid second messenger, phosphatidylinositol 3-phosphate. Proc. Natl Acad. Sci. USA 97, 8910-8915 (2000).

24. Feng, S. et al. A rapidly reversible chemical dimerizer system to study lipid signaling in living cells. Angew. Chem. Int Ed. Engl. 53, 6720-6723 (2014).

25. Balla, T. Phosphoinositides: tiny lipids with giant impact on cell regulation. Physiological Rev. 93, 1019-1137 (2013).

26. Luo, X. et al. Structure of the Legionella virulence factor, SidC reveals a unique $\mathrm{PI}(4) \mathrm{P}$-specific binding domain essential for its targeting to the bacterial phagosome. PLoS Pathog. 11, e1004965 (2015).

27. Tang, T. X., Finkielstein, C. V. \& Capelluto, D. G. S. The C-terminal acidic motif of Phafin2 inhibits $\mathrm{PH}$ domain binding to phosphatidylinositol 3-phosphate. Biochim Biophys. Acta Biomembr. 1862, 183230 (2020).

28. Nakatsu, F. et al. PtdIns4P synthesis by PI4KIIIalpha at the plasma membrane and its impact on plasma membrane identity. J. Cell Biol. 199, 1003-1016 (2012).

29. Shen, Y., Rosendale, M., Campbell, R. E. \& Perrais, D. pHuji, a pH-sensitive red fluorescent protein for imaging of exo- and endocytosis. J. cell Biol. 207, 419-432 (2014).

30. Taylor, M. J., Perrais, D. \& Merrifield, C. J. A high precision survey of the molecular dynamics of mammalian clathrin-mediated endocytosis. PLoS Biol. 9, e1000604 (2011).

31. Barger, S. R. et al. Membrane-cytoskeletal crosstalk mediated by myosin-I regulates adhesion turnover during phagocytosis. Nat. Commun. 10, 1249 (2019). 
32. Brzeska, H., Koech, H., Pridham, K. J., Korn, E. D. \& Titus, M. A. Selective localization of myosin-I proteins in macropinosomes and actin waves. Cytoskeleton (Hoboken) 73, 68-82 (2016).

33. Leyden, F. et al. Racl activation can generate untemplated, lamellar membrane ruffles. BMC Biol. 19, 72 (2021).

34. Yao, L. et al. Pleckstrin homology domains interact with filamentous actin. J. Biol. Chem. 274, 19752-19761 (1999).

35. Guntas, G. et al. Engineering an improved light-induced dimer (iLID) for controlling the localization and activity of signaling proteins. Proc. Natl Acad. Sci. USA 112, 112-117 (2015).

36. Putyrski, M. \& Schultz, C. Protein translocation as a tool: the current rapamycin story. FEBS Lett. 586, 2097-2105 (2012).

37. Ramirez, C., Hauser, A. D., Vucic, E. A. \& Bar-Sagi, D. Plasma membrane V-ATPase controls oncogenic RAS-induced macropinocytosis. Nature 576, 477-481 (2019).

38. Cerami, E. et al. The cBio cancer genomics portal: an open platform for exploring multidimensional cancer genomics data. Cancer Discov. 2, 401-404 (2012).

39. Gao, J. et al. Integrative analysis of complex cancer genomics and clinical profiles using the cBioPortal. Sci. Signal. 6, pl1 (2013).

40. Shin, H. W. et al. An enzymatic cascade of Rab5 effectors regulates phosphoinositide turnover in the endocytic pathway. J. Cell Biol. 170, 607-618 (2005).

41. Welliver, T. P. \& Swanson, J. A. A growth factor signaling cascade confined to circular ruffles in macrophages. Biol. open 1, 754-760 (2012).

42. He, K. et al. Dynamics of phosphoinositide conversion in clathrin-mediated endocytic traffic. Nature 552, 410-414 (2017).

43. He, K. et al. Dynamics of Auxilin 1 and GAK in clathrin-mediated traffic. J Cell Biol. 219, e20190814 (2020).

44. Boulant, S., Kural, C., Zeeh, J. C., Ubelmann, F. \& Kirchhausen, T. Actin dynamics counteract membrane tension during clathrin-mediated endocytosis. Nat. Cell Biol. 13, 1124-1131 (2011).

45. Crevenna, A. H. et al. Side-binding proteins modulate actin filament dynamics. eLife 4,e04599 (2015).

46. Hansen, S. D. et al.alphaE-catenin actin-binding domain alters actin filament conformation and regulates binding of nucleation and disassembly factors. Mol. Biol. cell 24, 3710-3720 (2013).

47. Bergert, M., Chandradoss, S. D., Desai, R. A. \& Paluch, E. Cell mechanics control rapid transitions between blebs and lamellipodia during migration. Proc. Natl Acad. Sci. USA 109, 14434-14439 (2012).

48. Henson, J. H. et al. Arp $2 / 3$ complex inhibition radically alters lamellipodial actin architecture, suspended cell shape, and the cell spreading process. Mol. Biol. cell 26, 887-900 (2015).

49. Le, A. H. et al. CYRI-A regulates macropinocytic cup maturation and mediates integrin uptake, limiting invasive migration. bioRxiv https://doi.org/ 10.1101/2020.12.04.411645 (2021).

50. Wu, C., Jin, X., Tsueng, G., Afrasiabi, C. \& Su, A. I. http://biogps.org/gene/ 79666. (2016).

51. Wu, C., Jin, X., Tsueng, G., Afrasiabi, C. \& Su, A. I. BioGPS: building your own mash-up of gene annotations and expression profiles. Nucleic acids Res. 44, D313-D316 (2016).

52. Campeau, E. et al. A versatile viral system for expression and depletion of proteins in mammalian cells. PloS One 4, e6529 (2009).

53. Steigemann, P. et al. Aurora B-mediated abscission checkpoint protects against tetraploidization. Cell 136, 473-484 (2009).

54. Raucher, D. et al. Phosphatidylinositol 4,5-bisphosphate functions as a second messenger that regulates cytoskeleton-plasma membrane adhesion. Cell 100, 221-228 (2000).

55. Oikawa, T., Itoh, T. \& Takenawa, T. Sequential signals toward podosome formation in NIH-src cells. J. Cell Biol. 182, 157-169 (2008).

56. Goedhart, J. et al. Structure-guided evolution of cyan fluorescent proteins towards a quantum yield of 93\%. Nat. Commun. 3, 751 (2012).

57. Ran, F. A. et al. Genome engineering using the CRISPR-Cas9 system. Nat. Protoc. 8, 2281-2308 (2013).

58. Hammond, G. R. et al. PI4P and PI $(4,5) \mathrm{P} 2$ are essential but independent lipid determinants of membrane identity. Science 337, 727-730 (2012).

59. Schneider, C. A., Rasband, W. S. \& Eliceiri, K. W. NIH image to ImageJ: 25 years of image analysis. Nat. methods 9, 671-675 (2012).

60. Schindelin, J. et al. Fiji: an open-source platform for biological-image analysis. Nat. methods 9, 676-682 (2012).

61. Sapoznik, E. et al. A versatile oblique plane microscope for large-scale and high-resolution imaging of subcellular dynamics. eLife 9, e57681 (2020).

62. Edelstein, A. D. et al. Advanced methods of microscope control using $\mu$ Manager software. J. Biol. Methods https://doi.org/10.14440/jbm.2014.36 (2014).

63. Schink, K. O. \& Bolker, M. Coordination of cytokinesis and cell separation by endosomal targeting of a Cdc42-specific guanine nucleotide exchange factor in Ustilago maydis. Mol. Biol. cell 20, 1081-1088 (2009).
64. Bigay, J., Casella, J. F., Drin, G., Mesmin, B. \& Antonny, B. ArfGAP1 responds to membrane curvature through the folding of a lipid packing sensor motif. EMBO J. 24, 2244-2253 (2005)

65. Tinevez, J. Y. et al. TrackMate: an open and extensible platform for singleparticle tracking. Methods 115, 80-90 (2017).

66. Schink, K. O. et al. The phosphoinositide coincidence detector Phafin2 promotes macropinocytosis by coordinating actin organisation at forming macropinosomes. https://doi.org/10.5281/zenodo.5512979 (2021).

\section{Acknowledgements}

We thank Dr. Frauke Ackermann for critical reading of the manuscript and useful discussions. Dr. Gareth Griffiths is acknowledged for useful discussions on macropinosome actin dynamics. We thank Ulrikke Dahl Brinch and Eva Rønning for expert technical support. The Advanced Light microscopy core facility and the Flow cytometry Core facility at Oslo University Hospital are acknowledged for providing access to relevant instruments. K.O.S was supported by a career development fellowship from the Norwegian Cancer Society, a Career grant from the South-Eastern Norway Regional Health Authority (2020038), and a Research Grant from the Research Council of Norway (315103). C.C. is a young investigator of the Research Council of Norway. H.S. was supported by an Open Project Grant from the South-Eastern Norway Regional Health Authority (2018081), a Research Grant from the Norwegian Cancer Society (182698), and an Advanced Grant from the European Research Council (788954). This work was partly supported by the Research Council of Norway through its Centres of Excellence funding scheme, project number 179571

\section{Author contributions}

K.O.S. and H.St. conceived and supervised the study. K.O.S. designed experiments, generated constructs, lentiviruses and stable cell lines, performed CRISPR/Cas9 knockouts, performed live cell and super-resolution microscopy, flow cytometry, lipid-binding assays, actin binding assays, wrote image and data processing software and analyzed data. K.W.T. generated constructs, lentiviruses and cell lines, performed live-cell imaging, and quantified data, H.Sp. performed image quantification and RNAi experiments. D.M. performed flow cytometry and protein-lipid interaction assays, generated constructs and cell lines. M.S. generated constructs and cell lines. C.C. generated constructs, cell lines and performed data analysis. V.S. and J.E. performed Salmonella infection assays. C.R. performed high content imaging and analysis. K.O.S. and H.St wrote the manuscript with input from all co-authors.

\section{Competing interests}

The authors declare no competing interests.

\section{Additional information}

Supplementary information The online version contains supplementary material available at https://doi.org/10.1038/s41467-021-26775-x.

Correspondence and requests for materials should be addressed to Kay Oliver Schink or Harald Stenmark.

Peer review information Nature Communications thanks Robert Kay and the other anonymous reviewer(s) for their contribution to the peer review of this work.

Reprints and permission information is available at http://www.nature.com/reprints

Publisher's note Springer Nature remains neutral with regard to jurisdictional claims in published maps and institutional affiliations.

\section{(i)}

Open Access This article is licensed under a Creative Commons Attribution 4.0 International License, which permits use, sharing, adaptation, distribution and reproduction in any medium or format, as long as you give appropriate credit to the original author(s) and the source, provide a link to the Creative Commons license, and indicate if changes were made. The images or other third party material in this article are included in the article's Creative Commons license, unless indicated otherwise in a credit line to the material. If material is not included in the article's Creative Commons license and your intended use is not permitted by statutory regulation or exceeds the permitted use, you will need to obtain permission directly from the copyright holder. To view a copy of this license, visit http://creativecommons.org/ licenses/by/4.0/.

(C) The Author(s) 2021 